hep-th/0507218

WIS/17/05-JUL-DPP

\title{
Constraining Modular Inflation in the MSSM from Giant Q-Ball Formation
}

\author{
Micha Berkooz ${ }^{a}$, Daniel J.H. Chung ${ }^{b}$ and Tomer Volansky ${ }^{a}$ \\ ${ }^{a}$ Weizmann Institute of Science, Rehovot, Israel \\ ${ }^{b}$ Department of Physics, University of Wisconsin, Madison, WI 53706
}

\begin{abstract}
We discuss constraints on which flat directions can have large vacuum expectation values (VEVs) after inflation. We show that only flat directions which are not charged under B-L and develop positive pressure due to renormalization group effects can have large VEVs of order $M_{\mathrm{Pl}}$. For example, within the MSSM only the $H_{u} H_{d}$ flat direction is found to be viable. This strongly constrains the embedding of a broad class of inflationary models in the MSSM or some other supersymmetric extension of the SM. For flat directions with negative pressure, the condensate fragments into very large Q-balls which we call Q-giants. We discuss the formation, evolution and reheating of these Q-giants and show that they decay too late. The analysis requires taking into account new phases of the flat directions, which have been overlooked in the formation and dynamics of the Q-balls. These constraints may be ameliorated by invoking a short period of thermal inflation. The latter, however, is viable in a very narrow window of parameter space and requires fine tuning.
\end{abstract}




\section{Introduction}

Inflation is a successful cosmological paradigm both from an observational point of view and theoretical point of view. It is observationally successful as the mounting cosmological data indicate that we live in a FRW universe with a flat spatial section and that density perturbations that seeded structure formation is adiabatic and nearly scale invariant. It is even partially falsifiable as a large class of inflationary models called "slow-roll" inflationary models may be ruled out through the consistency condition [1] if we are successful in measuring B-mode polarization which contains information about primordial gravity waves. From a theoretical point of view, inflation is appealing because it is the only known way to honestly solve the flatness problem of cosmology. Furthermore, because the energy scale of its physics may be as high as $10^{16} \mathrm{GeV}$, fundamental theories such as string theory may have a good chance of making contact with its observable physics.

Unfortunately, inflation does not have any smoking gun signatures yet because it has not made any connection with controlled experimental settings such as terrestrial colliders. Furthermore, there are no compellingly beautiful models of inflation as the constraints from the requisite large number of e-foldings, the small amplitude of density perturbations, and the smallness of the departure of the spectral index from scale invariance necessitate unnaturally tuned parameters in ad hoc constructions of inflationary models. The best hope for discovering whether inflation has occurred in our universe's history seems to be in finding a natural inflaton candidate in physics beyond the standard model (SM) discovered at future colliders.

One of the best motivated ideas for physics beyond the SM is supersymmetry (SUSY), and in particular, signatures of the minimal supersymmetric standard model (MSSM) are actively sought after in the next generation of high energy physics experiments such as the LHC (for a recent review in this direction, see for example, 2]). Furthermore, engineering required to construct successful inflationary scenarios is often more natural in the context of supersymmetry. Among the plethora of scalars afforded by supersymmetrizing the SM, flat directions are particularly enticing candidates for inflatons. ${ }^{1}$ Finally, from a perturbative string theory point of view, one of the simpler embeddings of the SM into the MSSM arises from gravity mediated SUSY breaking context. Hence, we are naturally led to ask which flat directions in the gravity mediated SUSY breaking scenario of MSSM may be part of the inflaton dynamics.

To this end, the story of Q-balls, a class of nontopological solitons [3, 4, 5, plays a crucial

\footnotetext{
${ }^{1}$ Throughout this paper, we collectively denote flat directions by $\phi$.
} 
role. Because nearly all of the flat directions in the MSSM have an accidental $U(1)$ symmetry at the renormalizable operator level, they fulfill one of the conditions for the existence of Q-balls (that of having a conserved global charge) 6, 7. The other crucial condition for the existence of Q-ball is that the scalar field potential $U$ satisfies

$$
\min _{\phi}\left[\frac{U}{|\phi|^{2}}\right]<\frac{1}{2} U^{\prime \prime}(0)
$$

This condition also has a good chance of being satisfied for MSSM flat directions because its interactions with the heavy degrees of freedom renormalize the mass (which is of the order of the SUSY breaking gravitino mass $\left.m_{3 / 2}\right)$ of the flat direction as $m_{\phi}^{2}\left[1+K \ln \left(\frac{|\phi|^{2}}{M^{2}}\right)\right]|\phi|^{2}$, and if $K<0$, Eq. (11) can be satisfied [8]. ${ }^{2}$

This story finds relevance in cosmology 9] because for situations in which a flat direction is displaced far from the origin at the end of inflation, one can show that when $K<0$, there is negative pressure [10, which generates the necessary instability for Q-ball formation. Furthermore, since the $U(1)$ is an accidental symmetry, non-renormalizable operators which break this $U(1)$ induce large charge in the condensate which ends up inside the Q-balls.

Therefore, as is known [8, 9, 11, 12, 13, 14, Q-balls generically form at the end of inflation if a flat direction is turned on during inflation and in particular, if it plays a role in the inflationary dynamics. It is also known that if the Q-balls are too large and survive certain violent destructive processes, they will live a long time before decaying, leading to a reheating temperature too low to be compatible with cosmology. Hence, certain MSSM flat directions can be ruled out as candidates for the inflaton (or more precisely, flat directions cannot be displaced far from the origin at the end of inflation) if the Q-balls form along these directions and live a long time.

We show that Q-balls with VEVs of order the Planck scale (which we call "Q-giants" due to their attendant large charge) generically live a long time, thus eliminating many flat directions from the possibility of being displaced far from the minimum at the end of inflation. The new physical observation that allows us to make this conclusion is that between the Q-giants, the density of gas of flat direction particles is necessarily large, thereby inducing large masses to all particles such that the decay rate of the gas is highly suppressed. Consequently, not only do the Q-giants live longer due to their suppressed decay rates, but also the thermal plasma produced by the decay of the gas is too cold to dissociate the Q-giants. Thus, all MSSM flat directions with negative value of $K$ are excluded as inflaton candidates. The only viable flat direction of

\footnotetext{
${ }^{2}$ Integrating out many heavy degrees of freedom during inflation is generic since the MSSM flat directions can be displaced far from the minimum, inducing large masses to the fields to which it couple.
} 
the MSSM that does not carry B-L charge is found to be $H_{u} H_{d}$. Finally, although B-L charge density carried by the effective Affleck-Dine field with VEV close to $M_{\mathrm{Pl}}$ is generically too large to be consistent with cosmology even when no Q-giants form, such directions may survive due to charge dilution through mechanisms such as thermal inflation (although as we discuss, constructing such thermal inflationary models will be contrived and requires fine tuning). Thus, $H_{u} L$ direction which has positive pressure may survive as an inflaton candidate.

Although most of the paper is concerned with the MSSM flat directions having $M_{\mathrm{Pl}}$ field variations (hence, from a string theory point of view, these would naturally be moduli fields), it is important to note that most of the results can be applied to other systems in which there is Q-giant formation starting from a field condensate. In particular, the constraints hold for flat directions in any extension of the SM, such as GUT or string embedding. Indeed, there are realizations of inflation where some of the moduli fields cannot be part of the MSSM, and should therefore arise from flat directions of some extension [15]. The results of this paper may strongly constrain a given extension. Furthermore, during (F-term) inflation, flat directions generically obtain corrections which may drive them alway from the origin [16, 17. The existence of such directions, even without playing a role in inflation, is highly constrained in the same manner.

The outline of the paper is the following. Section 2 contains an executive summary of the cosmological scenario and the results. Section 3 contains a discussion of the framework and some of the models of inflation to which it applies. Sections 4 and 5 discuss the case of negative pressure flat directions. Section 4 deals with the formation of Q-giants after inflation and section 5 deals with their subsequent evolution leading to too low of a reheat temperature to be compatible with cosmology. In section [6 we discuss positive pressure flat directions and show that they are cosmologically acceptable. Section $\square$ deals with thermal inflation as a possible loophole to our results. We argue that such a scenario however requires fine tuning and is very restricted. We conclude in section 8

We will use the notation $M_{\mathrm{Pl}} \equiv 1 / \sqrt{8 \pi G_{N}}=2.4 \times 10^{18} \mathrm{GeV}$ throughout the paper.

\section{The Cosmological Scenario: An Executive Summary}

In this section, we briefly outline the cosmological scenario and the main results of this paper.

We study inflationary models in the framework of gravity mediated supersymmetry breaking. In many natural models of inflation an inflationary epoch is induced with the use of moduli 
whose VEVs are $M_{\mathrm{Pl}}$ away from the true minimum and which dominate the energy density during inflation. A canonical example is hybrid inflation [18, 15, 19, 20, 21, 22. Such moduli are usually related to flat directions in a moduli space of a supersymmetric theory. The purpose of this paper is to investigate the dynamics of such flat directions and thus place constraints on their possible role in inflation. Furthermore, in many theories flat directions are driven away from the origin due to Hubble-induced couplings [16, 17. The existence of such flat directions may again be constrained. We concentrate on the MSSM, but stress that the analysis holds for any supersymmetric model with gravity mediated SUSY breaking.

\subsection{Assumptions}

Let us spell out the basic assumptions for which our scenario holds:

- At the end of inflation, the energy density is dominated by a flat direction, $\phi$, which is completely flat in the supersymmetric limit, $m_{3 / 2} \rightarrow 0$. As a consequence its potential is proportional to $m_{3 / 2}^{2}$ and is therefore of the form,

$$
U(\phi)=m_{3 / 2}^{2} M_{\mathrm{Pl}}^{2} U\left(\frac{|\phi|^{2}}{M_{\mathrm{Pl}}^{2}}, \frac{\phi^{n}}{M_{\mathrm{Pl}}^{n}}\right) .
$$

- One consequence of the above potential is that $\phi$ generically begins its evolution at $M_{\mathrm{Pl}}$.

- Another consequence is an approximate $U(1)$ for $\langle\phi\rangle \ll M_{\mathrm{Pl}}$. Thus at the onset of its evolution $\phi$ is "side-kicked" such that it carries an order one charge which is later released to the light particles including those of the MSSM. An immediate conclusion is that $\phi$ cannot carry B-L charge [19] with reheating temperature above the sphaleron transition temperature except under certain exceptional situations. ${ }^{3}$

\subsection{Cosmological Scenario}

Let us now point out the main features of the cosmological evolution that follows from the above assumptions. After taking into account renormalization group (RG) corrections to the modulus potential, the latter takes the form [8, 11],

$$
U(\phi)=m_{\phi}^{2}\left[1+K \log \left(\frac{|\phi|^{2}}{M^{2}}\right)\right]|\phi|^{2}+\sum_{k=1}^{\infty} \frac{m_{3 / 2}^{2} \phi^{n_{k}}}{M_{\mathrm{Pl}}^{n_{k}-2}}+\text { c.c. }
$$

\footnotetext{
${ }^{3}$ More on this point and its caveats in section 7
} 
where $m_{\phi} \simeq m_{3 / 2}, M$ is the scale at which $m_{3 / 2}$ is defined, typically the GUT or Planck scale and $K$ is related to the renormalization group beta function. $K$ can be negative or positive and typically ranges between $10^{-1}$ to $10^{-2}$. The values of $n_{k}$ depend on the symmetries (possibly discrete R-symmetries) of the model. Initially the condensate oscillates and due to the RG corrections positive or negative pressure is built up, depending on the sign of $K$ [10]. Before we present our computation, we summarize the $\phi$ field evolution in each of the two cases as follows:

Negative Pressure, $K<0$

- Negative pressure fragments the coherent condensate into very large Q-balls (Q-giants) and non-coherent, non-thermal particles with typical momentum of order $m_{\phi}^{-1}$.

- While almost all of the initial charge is stored in Q-giants, both the Q-giants and the $\phi$ gas initially store comparable amount of energy.

- Q-giant collisions break the giants into either smaller Q-balls or into the non-coherent gas. We show that while the gas comes to dominate the energy density rather quickly, at least a percent is left in the Q-giants.

- Outside the Q-giants $\langle\phi\rangle=0$, but

$$
\left\langle\phi^{\dagger} \phi\right\rangle \simeq 10^{-2}|K|^{2} M_{\mathrm{Pl}}^{2}
$$

This finite density effect induces large masses to any fields which are directly coupled to $\phi$. As $\phi$ decays through theses heavy particles, its decay rate is suppressed by $1 /\left\langle\phi^{\dagger} \phi\right\rangle$.

- The gas reheats the light particles outside the Q-giants to intermediate temperature,

$$
T_{\text {inter }} \simeq 10^{5} \mathrm{GeV}
$$

This temperature is too low to dissociate the Q-giants, and the smaller than naively expected temperature is due to the decay rate being suppressed by $1 /\left\langle\phi^{\dagger} \phi\right\rangle$.

- At this stage the temperature outside (for example, of the MSSM) dominates the energy density. However, since the Q-giants still hold at least a percent of the energy density in the form of non-relativistic matter, there is sufficient time for them to overcome the thermal radiation and dominate the energy density a long time before they decay, reheating to,

$$
T_{\text {reheat }} \simeq 10^{-4} \mathrm{GeV} .
$$




\section{Positive Pressure}

- In such cases, no fragmentation occurs.

- Due to the positive pressure, a finite Jeans scale develops, preventing the growth of small perturbations.

- By the time the evolution of the perturbations become non-linear, the Jeans scale is large enough to prevent the formation of primordial black holes (BHs). Therefore, there is no significant cosmological constraint coming from overproduction of BHs.

\subsection{Conclusions}

The above dynamics strongly constrains the possible use of flat directions in inflation:

- Negative pressure flat directions cannot develop VEVs of order $M_{\mathrm{Pl}}$ for viable cosmology. They are excluded since they decay very late, dominating the energy density during nucleosynthesis thus destroying its predictions. Furthermore, if charged under B, they release too much baryon number which cannot be erased at such low temperature.

- Positive pressure flat directions do not have the above problems, and further solve the problem of extensive formation of primordial BHs.

- Without invoking thermal inflation [23, 24, 25, B-L directions that either carry B or which reheat to temperatures high enough to produce B from sphaleron transitions are excluded due to excess baryon production. In section 7 we stress the difficulties in writing a model which incorporates a period of thermal inflation.

- For the case of flat directions in the MSSM, the only positive pressure flat direction which is not charged under B-L is $H_{u} H_{d}$. Hence within the MSSM and excluding exceptional situations presented in section [7 this is the only possible direction which can play a role in hybrid inflation or any other modular inflation.

- More generally, flat directions obtain Hubble-induced contributions to their masses during inflation. Such contributions drive them towards or away from the origin depending on the sign of the mass. Hence $H_{u} H_{d}$ is the only exactly flat direction in the MSSM which can obtain negative contributions of this kind. 


\section{The Framework}

One way of classifying models of inflation is by considering whether or not the inflaton field varies a field distance of order $M_{\mathrm{Pl}}$ during inflation. This classification has phenomenological utility as it can tell us about whether significant gravitational waves are generated or not 26]. Theoretically, large field variations signal theories with symmetries as potentials must be extremely flat to generically allow such large field variations without costing excessive energy. For example, in the context of perturbative string theory, Planckian field variations are natural, as the most ubiquitous scale in this theory is the string scale, and supersymmetry and modular invariance protect flat potential directions. As we will see, $M_{\mathrm{Pl}}$ field variations are also natural in hybrid inflationary models embedded in supergravity.

The results of our present paper regarding the formation and longevity of Q-giants are likely to apply to most scenarios of inflation in which the inflaton field at the end of inflation must still travel Planckian field values to reach the current minimum, and if the potential for this field satisfies the condition for Q-ball formation. The reasons is of course because the defining characteristic of a Q-giant, large Q-charge, is due to the large field displacement from the potential minimum. Therefore, even though we devote most of our attention in this paper with the end of inflation in the context of MSSM flat directions having Planckian field displacements, in the next subsection, we discuss more generally possible inflationary models where our results might be applicable. Note that although non-renormalizable operators are generically important for the inflationary scenarios with (near-)Planck field variation, the Qgiant analysis is insensitive to the details of the non-renormalizable operators.

\subsection{Applicable models}

There are many models of inflation that have been proposed which can involve Planck scale field variations. Arguably, most popular models of such type are chaotic inflationary scenarios 27] and hybrid inflationary scenarios [18, 28. Typically, for chaotic inflationary scenarios, the field variation is of order $M_{\mathrm{Pl}}$ during inflation and after inflation. On the other hand, hybrid inflation can involve field variations that are very small during inflation while the field variation is large after the end of inflation.

In the context of SUSY, the generic structure of SUSY inflation comes from the scalar 
potential

$$
U=\frac{1}{2} \Re\left[f_{a b}^{-1}\right] D_{a} D_{b}+e^{K / M_{\mathrm{Pl}}^{2}} g^{i j *}\left(D_{i} W\right)\left(D_{j} W\right)^{*}-\frac{3}{M_{\mathrm{Pl}}^{2}} e^{K / M_{\mathrm{Pl}}^{2} W^{*} W}
$$

where

$$
\begin{gathered}
g_{i j} \equiv \partial_{\phi_{i}} \partial_{\phi_{j}^{*}} K \\
D_{i} W=\frac{\partial W}{\partial \phi_{i}}+\frac{1}{M_{\mathrm{Pl}}^{2}} \frac{\partial K}{\partial \phi_{i}} W
\end{gathered}
$$

and where the D-terms in the flat space limit look like

$$
D_{a} \sim g \xi_{a}-g\left(\phi_{i}^{*} T_{i j}^{a} \phi_{j}\right)
$$

with $f_{a b}$ being the gauge kinetic function and $\xi_{a}$ not necessarily vanishing for a $U(1)$. For $U \neq 0$, if $D_{a} \neq 0$, we have D-term inflation [29, 20, 21], while otherwise, we have F-term inflation for which $W \neq 0$ or $D_{i} W \neq 0$.

F-term models typically suffer from the $\eta \equiv U^{\prime \prime} M_{\mathrm{Pl}}^{2} / U \geq 1$ problem (the $\eta$ problem) [28] which arises from the $e^{K / M_{\mathrm{P} 1}^{2}}$ factor. Although the D-term models typically do not suffer from this problem (since the F-term is zero by construction), in the context of string theory, if $g \xi_{a}$ comes from an anomalous $U(1)$, its value is generically too large to be compatible with cosmology. Note that even in D-term models the $\eta$ problem can arise since

$$
\frac{1}{2} \Re\left[f_{a b}^{-1}\right] D_{a} D_{b} \sim \xi^{2} g^{2}\left(1+\frac{\sigma^{2}}{\Lambda^{2}}+\ldots\right)
$$

which contributes

$$
\eta \sim \frac{\xi^{2} M_{\mathrm{Pl}}^{2}}{V \Lambda^{2}} \frac{g^{2}}{8 \pi}
$$

which would generically be too large for inflation to take place [30. However, this problem can be cured by imposing appropriate discrete symmetries.

D-term inflation typically realizes hybrid inflationary scenarios while F-term inflationary scenarios can realize both hybrid and chaotic scenarios. Because chaotic inflationary scenarios generically require the mass of the inflaton to be of order $10^{11} \mathrm{GeV}$, these directions are generically heavier than MSSM flat directions (i.e. this scenario is largely irrelevant for embedding MSSM flat directions into an inflationary scenario). Furthermore, although this scenario naturally will have Q-ball production, it gives rise to sufficiently large reheating temperature for a viable cosmology. Hence, we will now focus on discussing the salient features of hybrid inflationary scenarios. 
A common hybrid inflationary potential parameterization is

$$
U(\sigma, \phi)=\kappa^{2}\left(M^{2}-\frac{\phi^{2}}{4}\right)^{2}+\frac{\lambda^{2} \phi^{2} \sigma^{2}}{4}+\frac{m^{2} \sigma^{2}}{2}+\frac{\gamma}{4 !} \sigma^{4}
$$

where $\sigma$ is the field direction that is rolling during inflation when $\phi=0$ and $\phi$ is the waterfall field direction that shuts off inflation at the end when $\sigma$ rolls down to $\sigma^{2}<\sigma_{f}^{2} \equiv 2 M^{2} \kappa^{2} / \lambda^{2}$. In the standard scenario, we have

$$
\kappa^{2} M^{4} \gg m^{2} \sigma^{2} \gg \gamma \sigma^{4}
$$

which implies that the constant energy term $\kappa^{2} M^{4}$ dominates the energy density during inflation. The dominance of the constant energy density is the defining characteristic of hybrid inflationary models. When combined with the slow roll conditions and number of e-fold constraint with $m=m_{3 / 2} \sim 1 \mathrm{TeV}$ and $M \equiv \beta M_{\mathrm{Pl}}$ (the latter says that field variations of the waterfall field $\phi$ are Planckian at the end of inflation), the phenomenologically allowed parameter ranges are

$$
\begin{array}{rcl}
\frac{1}{\lambda}<\frac{1}{\sqrt{24 \pi^{2}}} \frac{\kappa^{2} \beta^{5}}{\sqrt{P_{\mathcal{R}}}}\left(\frac{M_{\mathrm{Pl}}}{m_{3 / 2}}\right)^{2} & \ll \beta \frac{M_{\mathrm{Pl}}}{m_{3 / 2}} \\
\sqrt{\gamma} \kappa^{3} \beta^{6} & \leq & 10^{-49} \\
\frac{m_{3 / 2}^{2}}{M_{\mathrm{Pl}}^{2}} & \ll \kappa^{2} \beta^{4} \ll & \frac{3}{8}(8 \pi)^{2} P_{\mathcal{R}} \sim 10^{-8} \\
\kappa^{2} \beta^{4} & \sim & \frac{m_{3 / 2}^{2}}{M_{\mathrm{Pl}}^{2}} \frac{N}{\ln \left(\frac{\sigma_{N}}{\sigma_{f}}\right)}
\end{array}
$$

where $P_{\mathcal{R}} \sim 10^{-9}$ is the adiabatic scalar perturbation power spectrum, $N \sim 50$ is the number of e-folds required for inflation, and

$$
\sigma_{N} \approx \frac{1}{2 \pi} \frac{1}{\sqrt{3 P_{\mathcal{R}}}} \frac{\left(\kappa^{2} M^{4}\right)^{3 / 2}}{M_{\mathrm{Pl}}^{3} m^{2}}
$$

is the field value $N$ e-folds before the end of inflation. ${ }^{4}$ The conditions given by Eqs. (15) and (16) can be associated with Eq. (14), while Eq. (17) comes from the standard slow roll conditions $\epsilon \equiv \frac{M_{\mathrm{Pl}}^{2}}{2}\left(U^{\prime} / U\right)^{2} \ll 1$ and $\eta \equiv M_{\mathrm{Pl}}^{2}\left(U^{\prime \prime} / U\right) \ll 1$.

A few remarks are now in order.

1. In the absence of symmetries, the parameters $\kappa, \lambda$, and $\gamma$ must be finely tuned.

\footnotetext{
${ }^{4}$ This is under the approximation that inflation really ends when $\sigma=\sigma_{f}$ which is only approximately true when the $\phi$ field is not too light.
} 
2. Within the context of supergravity, the waterfall field, $\phi$, is $M_{\mathrm{Pl}}$ away from its minimum at the end of inflation [15]. This has two consequences: (i) $\phi$ is weakly coupled to the MSSM and therefore suffers from a moduli problem [19]. One solution to this problem is to let the true minimum (as well as the origin of field space) be an enhanced symmetry point in which case the decay width of $\phi$ is large. (ii) By the time $\phi$ decays, $\sigma$ has mass of order the Planck scale.

3. If the parameterization of Eq. (13) is used, the waterfall field $\phi$ rolls away from the origin at the end of inflation. As noted by [15], this is one of the main reasons why it is difficult to identify one of the MSSM flat directions with $\phi$ : MSSM flat directions have VEVs that are zero or much smaller than $M_{\mathrm{Pl}}$ today. One may naively think it is easy to shift the origin of the inflationary potential. However, this is incorrect since a vacuum shift generically breaks the underlying symmetries governing the form of the original potential. Nevertheless, it could well be, if one is to solve the moduli problem, that the light MSSM fields do live away from the origin on another enhanced symmetry point. While common in string theory, such theories are complicated to write down, since the symmetries cannot be realized linearly. One should therefore think about the above Lagrangian as an expansion of the full theory around the origin, and can thus view $\phi$ as an MSSM field rolling away from the origin, and into the MSSM enhanced symmetry point.

As a concrete example in which $\phi$ is rolling away from the origin, let us first consider one of the the models of [15] (for improvements on this type of model and further discussion see [19]). They use the superpotential

$$
W=g \frac{\sigma^{2} \phi^{2}}{2 M_{\mathrm{Pl}}}
$$

(where $g$ is a coupling constant) and an instanton induced soft breaking terms with scales which are natural for gravity mediation SUSY breaking scenario. The resulting potential (neglecting the SUGRA corrections) can be written approximately as

$$
U(\phi, \sigma) \approx \frac{3}{4} \frac{\alpha^{2} m_{3 / 2}^{2}}{M_{\mathrm{Pl}}^{2}}\left(\frac{|\phi|^{2}}{4}-\beta^{2} M_{\mathrm{Pl}}^{2}\right)^{2}+m_{3 / 2}^{2}|\sigma|^{2}+\frac{g^{2}}{M_{\mathrm{Pl}}^{2}}\left(|\phi|^{4}|\sigma|^{2}+|\sigma|^{4}|\phi|^{2}\right)
$$

which can be identified with Eq. (13) through $\kappa \sim \alpha m_{3 / 2} /\left(M_{\mathrm{Pl}}\right), \gamma \sim g^{2}|\phi|^{2} / M_{\mathrm{Pl}}^{2}$, and $\lambda \sim$ $g^{2}\left|\sigma_{N}\right|^{2} / M_{\mathrm{Pl}}^{2}$. Note that this choice of $\kappa$ with $\alpha \sim 1 / \beta$ is natural for a flat direction field $\phi$ in supersymmetry. With this parameterization, because $\sigma$ is rolling towards smaller values during inflation, the condition $\sigma_{N} \sim 10^{4} \beta^{3} m_{3 / 2} \gg \sigma_{f} \sim m_{3 / 2} / \lambda$ must be satisfied. Hence, $\beta$ cannot be too small to inflate sufficient number of e-folds, which means, as we advertised, that Planck scale field variation of $\phi$ is natural in the context of SUSY hybrid inflation [19]. 
For an example of a model in which the waterfall field ends up at $\phi=0$, consider the model of [29]. This model has the merit in that it also solves the $\eta$ problem. The supersymmetric part of the model is defined by the superpotential

$$
W=\lambda_{1} \sigma \chi \psi_{1}+\lambda_{2} \frac{\phi^{n-2}}{M_{\mathrm{Pl}}^{n-2}} \phi^{2} \psi_{2}
$$

and a D-term

$$
D=\Lambda^{2}-|\chi|^{2}-|\phi|^{2}+\left|\psi_{1}\right|^{2}+n\left|\psi_{2}\right|^{2} .
$$

If $|\chi|^{2}+|\phi|^{2} \leq \Lambda^{2}$, the potential is minimized for $\psi_{1}=\psi_{2}=0$. Hence, ignoring for the moment the soft terms, the inflationary scalar potential becomes

$$
U=\lambda_{1}^{2}|\sigma|^{2}|\chi|^{2}+\lambda_{2}^{2} \frac{|\phi|^{2 n-4}}{M_{\mathrm{Pl}}^{2 n-4}}|\phi|^{4}+\frac{g^{2}}{2}\left(\Lambda^{2}-|\chi|^{2}-|\phi|^{2}\right)^{2}
$$

which inflates while $\sigma$ is large enough

$$
|\sigma| \geq \sigma_{f}^{\prime} \equiv \frac{\sqrt{n} \lambda_{2}}{\lambda_{1}}\left(\frac{\Lambda}{M_{\mathrm{Pl}}}\right)^{n-2} \Lambda
$$

with $\chi=0$ and the waterfall field pinned at

$$
|\phi|^{2} \approx \Lambda^{2}-n \frac{\lambda_{2}^{2}}{g^{2}}\left(\frac{\Lambda}{M_{\mathrm{Pl}}}\right)^{2 n-2} M_{\mathrm{Pl}}^{2} .
$$

Here we have expanded to leading order in $\lambda_{2}$.

During inflation, and taking into account soft breaking terms, the effective potential is of the form

$$
U=\lambda_{2}^{2} \frac{\Lambda^{2 n-4}}{M_{\mathrm{Pl}}^{2 n-4}} \Lambda^{4}+m_{3 / 2}^{2} \sigma^{2} .
$$

In the parameterization of Eq. (13), this model can be identified with $M \sim \Lambda$ and $\kappa \sim$ $\lambda_{2}\left(\Lambda / M_{\mathrm{Pl}}\right)^{n-2}$. Hence, to satisfy CMB fluctuation amplitude, one find using Eqs. (19) and (25), $\Lambda \sim M_{\mathrm{Pl}}$ and $\lambda_{2} \simeq m_{3 / 2} / M_{\mathrm{Pl}}$, giving $U \sim m_{3 / 2}^{2} M_{\mathrm{Pl}}^{2}$ during inflation.

After the end of inflation, we have $\chi$ rolling out to $\Lambda$ while $\phi$ rolls to 0 . Note however, that due to the D-term, $\phi$ is not a flat direction of the full gauge group. Thus, if one is to embed the MSSM in this model, one must introduce an additional $U(1)$ gauge group. As a final remark, we point out that the solution of [29] to the $\eta$-problem involves having $W=W_{\phi}=0$ which means that $\phi$ is F-flat. Thus these class of models may incorporate flat directions of the MSSM or any other supersymmetric extension.

In all of these models, there exists a global $U(1)$ which is important for the formation of Q-balls as we later explain. We will see in section 3.2. these accidental $U(1)$ symmetries are generic in MSSM flat directions. 


\subsection{A Specific Example - Flat Directions in the MSSM}

Flat directions are ubiquitous in supersymmetric theories. In particular there are numerous such directions in the MSSM, as was systematically found in [31, 17. As discussed in the introduction, our goal is to constrain the embedding of moduli in models of inflation in the MSSM and more generally the existence of flat directions in models which involve an inflationary epoch. One such restriction was explained in [19]. Indeed, if we are in a situation where all the energy in the universe after inflation is stored in some field with large VEV, then this field cannot carry B-L number, or else too much baryon asymmetry is generated. This kind of reasoning assumes, of course, no fine tuning, ${ }^{5}$ although the amount of this fine tuning is not clear once fluctuations are taken into account, due to processes similar to tachyonic preheating. Thus we are left with only five possible flat directions for the waterfall field:

\begin{tabular}{c|r} 
& $B+L$ \\
\hline \hline$H_{u} H_{d}$ & 0 \\
$Q \bar{u} L \bar{e}$ & 0 \\
$Q \bar{u} Q \bar{d}$ & 0 \\
$Q Q Q L$ & +2 \\
$\bar{u} \bar{u} \bar{d} \bar{e}$ & -2
\end{tabular}

Further constraints on the embedding may be pursued by investigating the cosmological evolution of such flat directions. Indeed it is known that under certain conditions (i.e. negative pressure and such flat directions being charged under some global $U(1)$ ), they fragment to form Q-balls. To see that such a $U(1)$ exists for the above directions, we note the following. In the MSSM, the above directions are flat at the level of the renormalizable superpotential, and one expects these flat directions to be lifted by non-renormalizable terms. However, in the models discussed in section 3.1 in order for the flat direction to be naturally $M_{\mathrm{Pl}}$ away from its minimum, it must be an exact flat direction in the supersymmetric limit [19]. This in particular means that soft breaking terms are proportional to $m_{3 / 2}^{2}$ [17. Hence assuming for simplicity only one flat direction, denoted by $\phi$, its potential is therefore,

$$
U(\phi)=m_{3 / 2}^{2} M_{\mathrm{Pl}}^{2} U\left(\phi^{n} / M_{\mathrm{Pl}}^{n},|\phi|^{2}\right) .
$$

\footnotetext{
${ }^{5}$ Finely tuned initial conditions could easily allow for an eccentric orbit which would suppress the produced baryon number.
} 
Thus as long as $\phi$ does not couple to light fields, ${ }^{6}$ it has an approximate $U(1)$ for $\phi / M_{\mathrm{Pl}}<1$. For the directions not charged under $\mathrm{B}$ or $\mathrm{L}$, this is no longer true once other fields become light. However, as we will see in section 4.3 with the exception of $H_{u} H_{d}$, this only occurs very late in the cosmological evolution, due to $\left\langle\phi^{\dagger} \phi\right\rangle$ condensate. For the $H_{u} H_{d}$ direction the above does not hold, due to the $\mu$-term which gives a mass to $\phi$ already in the supersymmetric

limit. Here we do not attempt to solve the $\mu$-problem and assume either $\mu$ is of order $m_{3 / 2}$ or it is promoted to a field as in the NMSSM 32. Due to the special properties of this direction (positive pressure), the absence of an approximate $U(1)$ is irrelevant and as we show below it is most suitable to play the roll of the modulus in inflation.

A remark is now in order. Above we have discussed the approximate $U(1)$ in the scalar sector. One might worry that outside Q-balls, $\phi$ may decay into fermions which remain light. However, as we show below by appealing to supersymmetry, since bosons which interact directly with $\phi$ become heavy, the fermions also becomes heavy and therefore the Q-balls remain stable. Hence below we assume that along the flat direction the interaction to lighter SM fields (to which it can decay) is through some particles whose mass is proportional to the VEV of $\phi$ or $\left\langle\phi^{\dagger} \phi\right\rangle$.

Flat directions are constrained even without driving inflation. Within the context of supergravity, flat directions obtain Hubble-induced corrections to their masses,

$$
U(\phi)=-c H_{I}^{2}|\phi|^{2}+\ldots
$$

during inflation [16, 17]. Here $H_{I}$ is the Hubble constant at inflation and $c$ is a model-dependent dimensionless parameter. If $c>0, \phi$ becomes tachyonic during inflation and is quickly driven away from zero to a minimum which, for exact flat directions, is $M_{\mathrm{Pl}}$ away. It then remains at this minimum until the end of inflation, when it starts rolling back towards the origin. This situation is constrained by the analysis below, much like the embedding of modular inflation.

\section{Negative Pressure Flat Directions - Formation of Q-Giants}

This section is devoted to analyzing the matter content right after the condensate fragments. As we show, the universe contains both Q-giants and a non-thermal distribution of low momenta $\phi$ particle. ${ }^{7}$ This will serve as an initial configuration for the system which we evolve through the reheating period in section 5 .

\footnotetext{
${ }^{6}$ Light fields means that these fields are not integrated out.

${ }^{7}$ This is in contrast with the thermal effects discussed in [33, 34].
} 
In the case of gravity mediated SUSY breaking, the potential along a flat direction is given by $[8,11,12,14$.

$$
U(\phi)=m_{\phi}^{2}\left[1+K \log \left(\frac{|\phi|^{2}}{M^{2}}\right)\right]|\phi|^{2}+\ldots
$$

where $M$ is the scale at which $m_{\phi} \simeq m_{3 / 2}$ is defined, typically the GUT scale and '...' denote non-renormalizable terms with $M_{\mathrm{Pl}}$ suppressed couplings. $K$ encodes the running of the mass of the flat direction and is determined by the RG equations (which we discuss at greater length in section (6), therefore it depends only on the low energy theory below the Planck scale.

As is well known (see e.g. [10]) and rederived in appendix $\mathbb{A}$, the above quantum corrections to the flat directions result with either positive or negative pressure, depending on the sign of $K$,

$$
\frac{P}{\rho}=\frac{K}{2+K}
$$

If negative, the condensate at the end of inflation fragments and forms Q-balls [8, 12, 35, 13, 14]. For the models we are interested in, this process happens relatively quickly after inflation, when the VEV of the modulus is of order the Planck scale. Since the charge of Q-balls is proportional to the square of the VEV, these large charged Q-balls can be called Q-giants which decay late, producing a low reheat temperature (and in some cases, too much baryon number). Thus negative pressure flat directions result with unacceptable cosmology and therefore cannot play the role of the inflationary moduli.

After reviewing some known properties of Q-balls in subsection 4.1, we show in 4.2 that the condensate fragments into Q-giants (whose statistics we compute) and a non-thermal distribution of $\phi$ particles. Section 4.3 discusses the properties of this gas.

\subsection{Q-Balls Properties}

Let us briefly summarize the basics of non-topological solitons, otherwise known as Q-balls [3, 4] (for a review see e.g. [14] and references therein). Consider a complex scalar field with a $U(1)$ invariant potential $U(|\phi|)$,

$$
\mathcal{L}=\left|\partial_{\mu} \phi\right|^{2}-U(|\phi|)
$$

The conserved charge is given by $Q=i \int d^{3} x\left(\dot{\phi}^{\dagger} \phi-\phi^{\dagger} \dot{\phi}\right)$. We choose an ansatz of the form,

$$
\phi=\phi(r) e^{i \omega t}
$$


under which the equation of motion (EOM), charge and mass become,

$$
\begin{gathered}
\frac{d^{2} \phi}{d r^{2}}+\frac{2}{r} \frac{d \phi}{d r}-\phi \frac{d U_{\mathrm{eff}}}{d \phi^{2}}=0, \\
Q=2 \omega \int d^{3} x \phi^{2}(r), \\
M_{Q}=\int d^{3} x\left[\left(\partial_{r} \phi\right)^{2}+U_{\mathrm{eff}}(\phi)\right]+\omega Q,
\end{gathered}
$$

with $U_{\text {eff }}(\phi)=U(\phi)-\omega^{2} \phi^{2}$. This EOM describes a particle moving in an inverted effective potential with friction $\propto 1 / t$. We seek a solution which minimizes the energy and with boundary conditions, $\phi^{\prime}(0)=\phi^{\prime}(\infty)=0$. A stable solution necessarily exists if $2 U\left(\phi_{0}\right) / \phi_{0}^{2}$ has a minimum away from the origin. This condition holds for the potential (30) for negative $K$, which is the case we are dealing with here (postponing positive $K$ to section 6).

Here and below, we assume $|K| \log |\phi|^{2} / M^{2} \ll 1$. This allows us to use the 1-loop approximation for the potential without having to sum over large logs. We will later see that given $K$ of order $10^{-2}$ this assumption is easily justified, at least at the onset of Q-ball formation.

The Q-ball solution relevant in gravity mediated SUSY breaking is the thick wall Q-ball solution [1]. The ansatz for this solution

$$
\phi(r)=\phi_{0} e^{-\frac{r^{2}}{R^{2}}}
$$

with $\phi_{0} \ll M_{\mathrm{Pl}}$ (so that we can ignore non-renormalizable terms). Plugging this ansatz in the EOM (34) we get,

$$
-\frac{6}{R^{2}}+\frac{4 r^{2}}{R^{4}}=-\left(\omega^{2}-m_{\phi}^{2}(1+K)\right)-\frac{2 K m_{\phi}^{2} r^{2}}{R^{2}}+K m_{\phi}^{2} \log \frac{\phi_{0}^{2}}{M^{2}} .
$$

Comparing terms one finds

$$
\begin{aligned}
R & \simeq \sqrt{\frac{2}{|K|}} m_{\phi}^{-1}, \\
\omega^{2} & \simeq m_{\phi}^{2}\left(1-2 K+K \log \frac{\phi_{0}^{2}}{M^{2}}\right),
\end{aligned}
$$

and using eqs. (35) and (36),

$$
\begin{aligned}
Q & =\left(\frac{\pi}{2}\right)^{3 / 2} 2 \omega \phi_{0}^{2} R^{3} \\
M_{Q} & \simeq 3\left(\frac{\pi}{2}\right)^{3 / 2} \phi_{0}^{2} R+\left(2-\frac{7}{2} K+2 K \log \frac{\phi_{0}^{2}}{M^{2}}\right)\left(\frac{\pi}{2}\right)^{3 / 2} m_{\phi}^{2} \phi_{0}^{2} R^{3}
\end{aligned}
$$


where we used $\omega \simeq m_{\phi}$ and $1 / R \ll m_{\phi}$.

Two remarks are now in order. First, note that eq. (39) requires that the Hubble radius is much larger than $m_{\phi}^{-1}$ for Q-balls to form. In particular, $H^{-1} / R \gg 1$ which leads to $\phi_{0} / M_{\mathrm{Pl}} \ll(3 K / 2)^{1 / 2}$. This requirement is consistent with neglecting the non-renormalizable terms. A second remark is that stability is guaranteed by the fact that $K<0$. This ensures that the effective mass of $\phi$ increases as the VEV of $\phi$ decreases and therefore

$$
\frac{M_{Q}}{Q} \simeq m_{\phi}\left(1-\frac{3}{4} K-\frac{3}{2} K \log \frac{\phi_{0}^{2}}{M^{2}}\right)<\lim _{\phi_{0} \rightarrow 0} \sqrt{U^{\prime \prime}(\phi)} .
$$

In particular, at $\phi_{0}=0$, further light degrees of freedom must be reintroduced (recall that the above potential is obtained by integrating out heavy particles). Therefore, $\phi_{0}$ in the logs should be replaced by the low energy scale which can be taken to be $m_{\phi} \simeq 10^{3} \mathrm{GeV}$. Since $K<0$, this implies that the mass of the free particle [given by the right hand side of Eq. (43)] is much larger than the mass per charge of the Q-ball, rendering these Q-balls stable.

\subsection{Formation of Q-Giants and $\phi$ Gas}

To predict the size and number of Q-giants, we will make two assumptions which are physically reasonable and generic. The first is that, assuming no fine tuning, the orbit of the flat direction is homogeneous on a Hubble patch with non-negligible $U(1)$ charge [19]. The second assumption is that in all the relevant length scales, initially, $\delta \phi_{i} / \phi_{i} \simeq H / M_{\mathrm{Pl}} \simeq 10^{-15}$ when $\phi_{i}$ is of order $M_{\mathrm{Pl}}$ and the dynamics we are focusing on begins. ${ }^{8}$

When the condensate fragments, not all particles in the condensate make it into the Qgiants. We will refer to the remnant particles as the $\phi$ gas.

Q-Giants: The charge (and therefore size) of these Q-balls is determined by the time at which the first perturbation goes non-linear [11, 14,

$$
H_{0} \simeq \frac{2|K| m_{\phi}}{\alpha}, \quad \alpha=-\log \left(\delta \phi_{i} / \phi_{i}\right)
$$

Given that $\phi$ dominates the energy density, we can estimate its value when the Q-balls are formed,

$$
\phi_{0}=\frac{\sqrt{3} H_{0} M_{\mathrm{Pl}}}{m_{\phi}} \simeq \frac{\sqrt{12}|K| M_{\mathrm{Pl}}}{\alpha}
$$

\footnotetext{
${ }^{8}$ Strictly speaking, the size of the fluctuation might grow somewhat by the time the field moves from a VEV $\geq M_{\mathrm{Pl}}$ to the regime of validity of this potential. This growth is expected to change the estimate of $H$ at the time of Q-ball formation by a multiplicative factor of order 1. In addition, such a process can only serve to increase $H$ which will result in an increase of the Q-giants size which will only make our bounds more stringent.
} 
and using eqs. (39) and (411) one obtains,

$$
Q=\frac{2 \pi^{3 / 2}}{|K|^{3 / 2}} \frac{\phi_{0}^{2}}{m_{\phi}^{2}} \simeq 10^{28}\left(\frac{m_{\phi}}{10^{3} \mathrm{GeV}}\right)^{-2}\left(\frac{K}{10^{-2}}\right)^{1 / 2}\left(\frac{\alpha}{30}\right)^{-2} .
$$

The validity of this estimate was verified numerically in [36, 13. Due to the large initial value of $\phi$, these Q-balls store an extraordinary amount of charge, hence the name Q-giants.

We stress that the $\phi$ field that we are using cannot serve as an Affleck-Dine (AD) field [37. 17. The reason is that it dominates the energy density (since it has the largest VEV possible in this model) and hence if it were the $\mathrm{AD}$ field, it would have generated a very large baryon asymmetry. Thus the baryon number must be generated either by another AD field or by a variant of EW-baryogenesis [38, 39, 40, 41, 42, 43, 44] if the radiation temperature to be calculated below is above the electroweak (EW) scale.

$\phi$-Gas: The charge in the $\phi$ condensate is maximal if the VEV moves in a circular orbit. In this case, all the charge makes its way efficiently into Q-giants. In the generic situation we are discussing, the orbit is not circular. We show below that in this case, in addition to Q-giants, a distribution of low momenta (non-coherent) $\phi$ particles appears. We will refer to this as the $\phi$-gas. The existence of such a component is also supported in numerical simulations of condensate fragmentation [13. The critical features of this gas are discussed in section 4.3 ,

\subsubsection{Efficiency of Q-Giants and Gas Formation}

Our initial configuration is that of a condensate, for which the perturbation on some scale grow to become non-linear. Once the non-linear regime is formed, Q-giants form very rapidly, as does the $\phi$ gas. Immediately after fragmentation the universe contains a mixture of the following components:

1. Energy in Q-giants - an order one fraction of the charge initially goes into Q-giants.

2. A gas of $\phi$ particles outside the Q-giants - the remaining energy goes initially into this gas.

3. Light MSSM particles which result from the decay of the $\phi$ particles - initially there are none of those. 
The physics of the fragmentation process is the following. The length scale that becomes non-linear first is of order 13

$$
\frac{k}{a} \sim R^{-1} \simeq\left(\frac{|K|}{2}\right)^{1 / 2} m_{\phi}
$$

where $a$ is the expansion factor. Once this mode enters the non-linear regime Q-giants form quickly, roughly one per the length scale (47). $\phi$ quanta that do not make it into Q-giants appear into a non-coherent gas of $\phi$ (and anti- $\phi$ ) particles. Since the entire process occurs for low momenta - of order $m_{3 / 2}$ up to some powers of $K$ - we expect that the $\phi$ gas will contain only low momenta. This picture is also verified numerically in [13. Furthermore, the same simulations suggest that most of the charge finds its way into the Q-giants rather than into the $\phi$ gas. $^{9}$

It is also easy to see that the amount of anti-Q-giants is very small. Indeed, recall that the condensate is rotating in one direction with a charge close to the maximal allowed. To get a Q-giant with the opposite charge, the condensate in a large piece of spacetime has to coherently start rotating the other way, which is suppressed. It is also unlikely that a Q-giant will form out of a gas of particles after the condensate fragmented. This is from phase space arguments: a non-coherent collection of $\phi$ quanta will not be able to assemble itself into a coherent Q-giant (or anti-Q-giant).

The total energy density in the $\phi$ condensate at the time of fragmentation is of order $\rho_{\text {total }}=m_{\phi}^{2} \phi_{0}^{2}$. The energy per charge in the condensate is given by $\rho_{\text {total }} / Q \simeq m_{\phi} /(1-\epsilon)$ where $\epsilon$ denotes the eccentricity of the orbit of $\phi$ and is therefore less than unity. On the other hand once Q-giants have formed, they fulfill $M_{Q} / Q \simeq m_{\phi}$ [see eqs. (41), (42)]. Thus since most of the charge ends up inside the Q-giants, the energy and charge density of the system's constituents after the Q-giant formation is

$$
\begin{array}{ll}
Q_{\text {gas }} / V \simeq 0 & \rho_{\text {gas }} \simeq \epsilon m_{\phi}^{2} \phi_{0}^{2} \\
Q / V \simeq(1-\epsilon) m_{\phi} \phi_{0}^{2} & \rho_{Q} \simeq(1-\epsilon) m_{\phi}^{2} \phi_{0}^{2}
\end{array}
$$

Finally, for consistency of this picture we need to show that the mode-mode interactions in the $\phi$ gas are small enough such that the gas does not thermalize but rather remains at low

\footnotetext{
${ }^{9}$ An intuitive argument may be the following. The VEV of the field inside the Q-giant moves in a circular orbit in field space. This means that regions in spacetime in which the charge-density/energy-density ratio is maximal have an easier time to fall into Q-giants. The $\phi$ gas originate from regions of space in which the chargedensity/energy-density ratio is low - such regions tend to be less coherent - but this also implies that the gas is characterized by a low charge-density/energy-density ratio.
} 
momenta until it decays. Indeed, there are only two kinds of interactions which are of importance to us: interactions arising from the log term in (30) and non-renormalizable interactions coming from corrections to the Kahler potential. For the latter, as discussed in section [3.2, a n-point interaction is suppressed by $m_{3 / 2}^{2} / M_{\mathrm{Pl}}^{n-2}$. Thus the rate for momentum transfer can be estimated to be

$$
\Gamma_{\mathrm{MM}}^{\mathrm{Non}-\mathrm{Ren}} \simeq n_{\phi}\left\langle\sigma_{\phi} v\right\rangle \lesssim \frac{|K|^{2} m_{3 / 2}^{3}}{M_{\mathrm{Pl}}^{2}},
$$

where $\sigma_{\phi}$ is the cross-section for these interactions and the $|K|^{2}$ factor comes from the suppression in the number density of the gas. Here we have estimated $\Gamma_{\mathrm{MM}}$ for $n=4$. Higher $n$ would result with lower rates.

The log interactions are more subtle. As we discuss at length in section 4.3, while $\langle\phi\rangle$ vanishes outside the $Q$-giants, the states which interact with $\phi$ remain massive due to nonvanishing $\left\langle\phi^{\dagger} \phi\right\rangle$. Thus the log potential is still valid outside the Q-giants and result with interactions suppressed by $\left\langle\phi^{\dagger} \phi\right\rangle$. Thus one finds

$$
\Gamma_{\mathrm{MM}}^{\mathrm{Log}} \lesssim \frac{|K|^{2} m_{3 / 2}^{3}}{\left\langle\phi^{\dagger} \phi\right\rangle}
$$

which is faster than the scattering rate eq. (49) and is of order the decay rate for $\phi$ (see section 4.3). In fact, we expect these scattering to be slower than the estimate above since 2-2 scattering (for which the estimation was done) cannot result with an increase of the average momenta while higher n-point scattering is further suppressed. We thus conclude that the gas outside the Q-giants is formed out of equilibrium and stays that way until it decays.

Finally, light MSSM particles are absent until the gas decays. We compute the gas decay rate in section 4.3 In section 5.2 we show that the temperature of the MSSM cannot dissociate the Q-giants.

\subsubsection{Initial Velocity and Charge Distribution in Q-Giants}

In section 5.1 we briefly investigate the consequence of Q-giant collisions. In order to do that, we must estimate the distribution of velocity and charge of the Q-giants. We do that here.

Let us denote a given Q-giant by its center $\vec{q}$ and radius $R$. The momentum current is then found to be

$$
P^{i}(\vec{q}, R)=\int_{V(\vec{q}, R)} d^{3} x a^{3}\left[\partial^{i} \phi^{\dagger} \partial^{0} \phi+\partial^{0} \phi^{\dagger} \partial^{i} \phi\right]
$$


where $V(\vec{q}, R)$ is the volume of the Q-giant and $x^{i}$ are co-moving spatial coordinates. To extract the distribution of momentum, we follow the evolution of $\phi$ until fluctuations become of order one, when $\phi=\phi_{0}$. Thus expanding around the homogeneous solution,

$$
\phi(x, t)=\phi(t)+\delta \phi(x, t)
$$

one finds to lowest order in $\delta \phi$,

$$
\left\langle\delta P^{i}\left(\vec{q}_{x}, R\right) \delta P^{j}\left(\vec{q}_{y}, R\right)\right\rangle \simeq|\dot{\phi}(t)|^{2} \int_{V_{x} V_{y}} d^{3} x d^{3} y a^{2} \frac{\partial}{\partial x^{i}} \frac{\partial}{\partial y^{j}}\left\langle\delta \phi^{\dagger}(x) \delta \phi(y)\right\rangle .
$$

The above can be estimated at the time of Q-giant formation by taking $\delta \phi \simeq \phi \simeq \phi_{0}$ and estimating the typical momentum to be of order $k \simeq R^{-1}$. Hence we get

$$
\delta P \simeq \omega \phi_{0}^{2} R^{2}
$$

which leads to the velocity distribution,

$$
\delta v \simeq \frac{\delta P}{M_{Q}} \simeq \sqrt{|K|}
$$

The charge distribution can be found in a similar fashion. Using the expression for $\mathrm{Q}$ given above, eq. (33), and expanding as in (52), one finds

$$
\left\langle\delta Q\left(\vec{q}_{x}, R\right) \delta Q\left(\vec{q}_{y}, R\right)\right\rangle \simeq|\phi|^{2} \int_{V_{x} V_{y}} d^{3} x d^{3} y a^{6} \frac{\partial}{\partial x^{0}} \frac{\partial}{\partial y^{0}}\left\langle\delta \phi^{\dagger}(x) \delta \phi(y)\right\rangle
$$

from which one can estimate,

$$
\delta Q \simeq \omega \phi_{0}^{2} R^{3}
$$

and therefore $\delta Q / Q \sim 1$.

Given the above, let us assume an initial Gaussian distribution ${ }^{10}$,

$$
f\left(t_{0}, Q, v\right)=N \frac{e^{-(Q-\langle Q\rangle)^{2} / 2 \delta Q^{2}}}{\left(2 \pi \delta Q^{2}\right)^{1 / 2}} \frac{e^{-v^{2} / 2 \delta v^{2}}}{\left(2 \pi \delta v^{2}\right)^{3 / 2}},
$$

with $\langle Q\rangle$ given in (41). Here $N$ is the normalization which can be approximated by assuming most of the charge is stored in the Q-giants [13,

$$
(1-\epsilon) m_{\phi} \phi_{0}^{2}=\int d Q Q \int d^{3} v f\left(t_{0}, Q, v\right)
$$

where $\epsilon$ is the initial eccentricity which is of order one (see eq. (48)). This gives,

$$
N \sim \frac{(1-\epsilon)}{R^{3}} \simeq(1-\epsilon) m_{\phi}^{3}|K|^{3 / 2}
$$

We will later use this distribution to estimate Q-giant destruction rates.

\footnotetext{
${ }^{10}$ This assumption is valid in the limit $\sqrt{K} \ll 1$ in which case there are many Q-giants (of order $10 / \sqrt{K}$ ) within a Hubble radius.
} 


\subsection{Stability of the $\phi$ Gas}

In the non-relativistic gas of $\phi$ particles, the relevant energy per particle are of order $m_{3 / 2}$. Hence during the stage of Q-giant formation, one must have, $\left\langle(\partial \phi)^{2}+U(\phi)\right\rangle=\rho_{\phi}$ which when combined with eqs. (30) and (48) gives

$$
\left\langle\phi^{\dagger} \phi\right\rangle=f_{\text {gas }} \phi_{0}^{2}
$$

where $f_{\text {gas }} \approx \epsilon$ at the onset of Q-giant formation and is roughly 1 by the time the gas decays due to energy loss of Q-giants through collisions (as discussed in section 5.1). On the other hand $\langle\phi\rangle \approx 0$ between the Q-giants.

We now argue the following:

1. All the states which are strongly coupled to $\phi$ are heavy $-m^{2} \sim\left\langle\phi^{\dagger} \phi\right\rangle$ - due to this large variance in the field and despite the fact that $\langle\phi\rangle=0$.

2. While giving large masses to all particles, $\phi$ itself remains light to all order of perturbation theory. This is a consequence of supersymmetry.

3. As a result, $\phi$ is stable and decays late, reheating the MSSM particles to temperature $T_{\text {inter }} \simeq m_{\phi}\left(M_{\mathrm{Pl}} / m_{\phi}\right)^{1 / 6}$. This tempertature, as discussed in section[5.2] cannot dissociate the Q-giants, which remain stable and dominate the energy density until they decay much later.

To get a sense for why the above is true, let us work with a specific model. As an example, consider the renormalizable interaction ${ }^{11}$

$$
W_{0}=\lambda \phi X^{2}+\ldots
$$

where $\lambda$ is an order one dimensionless coupling and '...' stands for additional non-renormalizable terms. This is the type of interaction we expect in the context of flat directions of the MSSM. The scalar potential then takes the form,

$$
U_{\text {scalar }}=4|\lambda|^{2}\left|\phi^{2}\right||X|^{2}+|\lambda|^{2}|X|^{4}+(\text { soft terms })+\ldots
$$

and there are Yukawa couplings,

$$
-L_{\text {Yukawa }}=2 \lambda \phi \Psi_{X} \Psi_{X}+\lambda X \Psi_{\phi} \Psi_{X}
$$




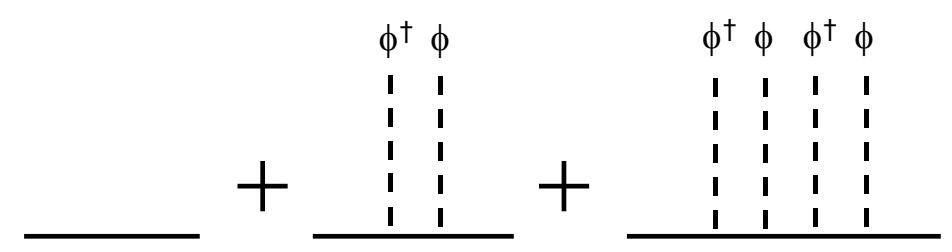

Figure 1: Diagrams contributing to the fermion's two-point functions.

where $\Psi_{\phi, X}$ are chiral fermions.

The first term in (63) gives a mass to $X, m_{X}^{2}=4|\lambda|^{2}\left\langle\phi^{\dagger} \phi\right\rangle$, thus making it very heavy. On the other hand, one may naively think that $\Psi_{X}$ remains light since it interacts with $\phi$ rather than $\phi^{\dagger} \phi$. To see that this is not the case, recall that the physical mass for a fermion is not $m$ which is charged under chiral rotations, but rather $|m|^{2}$ which in that case will be proportional to $\left\langle\phi^{\dagger} \phi\right\rangle$. Another way to see this is to look in the limit of exact supersymmetry (assuming $\left\langle\phi^{\dagger} \phi\right\rangle$ does not directly break SUSY $)^{12}$. In that limit $X$ and $\Psi_{X}$ must have similar masses and therefore the fermion must be massive as well. To be more concrete, let us compute the new propagator for $\Psi_{X}$ in the presence of non-vanishing $\left\langle\phi^{\dagger} \phi\right\rangle$. Summing over the diagrams in Fig. 1 one finds the corrected propagator,

$$
\left\langle\Psi_{X} \Psi_{X}\right\rangle=\frac{\not p}{p^{2}-4|\lambda|^{2}\left\langle\phi^{\dagger} \phi\right\rangle} .
$$

Indeed, the pole is at $4|\lambda|^{2}\left\langle\phi^{\dagger} \phi\right\rangle$ which is just $m_{X}^{2}$.

One may also worry that thermal and plasma effects induce a large splitting between the masses of $X$ and $\Psi_{X}$ which contributes to the mass of $\phi, m_{\phi}^{2} \propto m_{X}^{2}-m_{\Psi_{X}}^{2}$. Again, this can be argued not to occur. Indeed, in the supersymmetric limit, as was argued above, there is no splitting between $X$ and $\Psi_{X}$ and therefore no contribution the $m_{\phi}$. This can also be seen, if one notices that the contribution of the bosonic diagrams to $m_{\phi}^{2}$ in the presence of $\left\langle\phi^{\dagger} \phi\right\rangle$ (Fig. 2a), cancel the contribution from the fermionic diagrams (Fig. 2b). Any supersymmetric breaking effect, arise either from soft breaking terms which are proportional to $m_{\phi} \sim m_{3 / 2}$ or from plasma effects (recall that the gas is not in thermal equilibrium and therefore one cannot assign a temperature to it) which must be of order $k \sim R^{-1}=\sqrt{|K| / 2} m_{\phi}$. Thus any splitting must also be of order $m_{3 / 2}$ and therefore $\phi$ remains light.

Having established a consistent picture, we may now ask when does the $\phi$ gas decay. Since

\footnotetext{
${ }^{11}$ Of course, if no renormalizable interactions exist and only $M_{\mathrm{Pl}}$ suppressed ones, the decay rate of the $\phi$ gas is very low

${ }^{12}$ It is important to distinguish between finite density effects (from $\left\langle\phi^{\dagger} \phi\right\rangle$ ) and finite temperature SUSY breaking effects.
} 


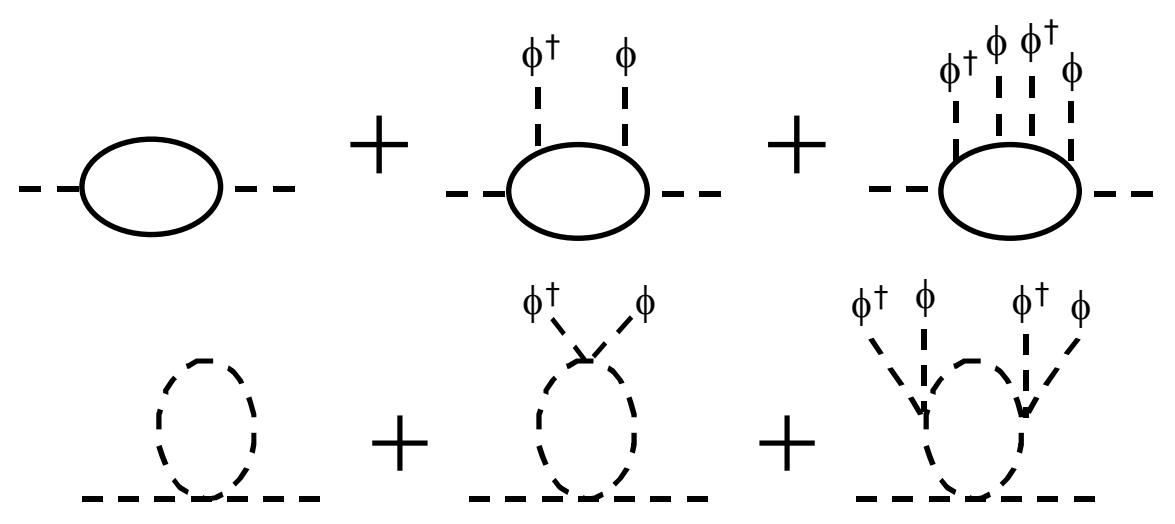

Figure 2: Contributions of bosons and fermions to $m_{\phi}^{2}$. In the supersymmetric limit, the bosonic contribution cancels the fermionic contribution exactly.

it is coupled to light degrees of freedom only through heavy particles of mass $\sim\left\langle\phi^{\dagger} \phi\right\rangle$, its decay rate can be bounded by

$$
\Gamma_{\phi} \leq \frac{m_{\phi}^{3}}{8 \pi\left\langle\phi^{\dagger} \phi\right\rangle}
$$

The energy densities in the gas and in the Q-giants are comparable and redshifts like $a^{-3}$. Thus we may assume the gas dominates the energy density and compute the intermediate reheating process by solving the equation for the energy density in the radiation,

$$
\dot{\rho}_{\gamma}+4 H \rho_{\gamma}=\Gamma_{\phi} \rho_{\phi}
$$

Using (44) and (66) and taking $H=2 /(3 t)$ for this matter dominated epoch, one can estimate,

$$
\rho_{\gamma} \simeq \frac{3}{11} \frac{f_{\text {gas }}}{8 \pi} m_{\phi}^{5} t
$$

This solution is valid until radiation becomes dominant at $H=\Gamma_{\phi}$. At this stage, the reheating ends and the gas decays completely, producing an intermediate temperature given by

$$
T_{\text {inter }} \simeq g_{*}^{-1 / 4} m_{\phi}\left(\frac{f_{\text {gas }} M_{\mathrm{Pl}}}{8 \pi m_{\phi}}\right)^{1 / 6} \simeq 10^{5} \mathrm{GeV}
$$

with the gas decaying when,

$$
\left.\left\langle\phi^{\dagger} \phi\right\rangle\right|_{\text {decay }} \simeq m_{\phi}^{2}\left(\frac{M_{\mathrm{Pl}}}{8 \pi f_{\text {gas }}^{1 / 2} m_{\phi}}\right)^{2 / 3} .
$$

Note that unlike the usual inflaton scenario, this intermediate reheating does not start at high temperature and therefore there is no danger to the stability of the Q-giants at this stage. 
From that point onwards, radiation surrounds the Q-giants. Initially it dominates the energy density but very quickly (once the expansion factor grows by two orders of magnitude) it becomes sub-dominant with Q-giants dominating until they decay. Note that taking into account sphalerons in the case where the charge in the giants corresponds to $\mathrm{B}+\mathrm{L}$, is not expected to change the final $\mathrm{B}+\mathrm{L}$ charge since the gas outside the Q-giants stores very little charge. Below we further investigate the dynamics of the Q-giants and show that the temperature of radiation does not dissociate the Q-giants.

\section{Evolution of Q-Giants and Reheating}

The evolution and stability of Q-balls has been widely investigated [45, 9, 11, 14, 46]. There are many effects which can influence the time of decay and the reheat temperature. We will consider the most relevant effects: fragmentation of Q-giants through collisions, dissociation due to external thermal plasma, and evaporation to bosons and fermions.

In addition, there is potentially a new effect which is the decay of Q-giants when immersed in the non-thermal gas of low momenta $\phi$ particle. However, as we saw above, in cases where the Q-giants are large the gas outside has large $\left\langle\phi^{\dagger} \phi\right\rangle \simeq f_{\text {gas }} \phi_{0}^{2}$ which suppresses interactions between the Q-giants and the $\phi$ gas at the surface (as well as with light particles).

\subsection{Q-Giant Collisions}

Q-giants may collide between themselves leading to charge and energy transfer and may be the cause of Q-giants disintegration. Collisions are especially important since the reheat temperature is inversely proportional to a power of the charge (see equation (92)), and hence if the Q-giants fragment to Q-balls with lower charge, the reheat temperature will be higher. We now show that Q-giant collisions only mildly reduce the number of Q-giants with large charge.

The only long range Q-giant/Q-giant interaction is gravitational. In addition they interact through direct collisions and interact with the gas around them. Here we focus on the effects of collisions. We expect that the cross-section for collision is of order the size of the Q-giant, which is verified in numerical simulations [46. More precisely, in general collisions are divided into three types: fusion, charge exchange and elastic. The cross-section for each is different with charge exchange being the largest, of order four times the geometric cross-section ${ }^{13}$. To

\footnotetext{
${ }^{13}$ This is the largest cross-section for a process that can reduce the charge. A larger cross-section can occur
} 
see how collisions reduce the number of Q-giants, let us define

$$
n_{Q}(t) \equiv \int_{\langle Q\rangle / 2}^{3\langle Q\rangle / 2} d Q^{\prime} \int d^{3} v f\left(t, Q^{\prime}, v\right)
$$

with $f(t, Q, v)$ is the distribution function in velocity $v$ and charge $Q$ of the Q-giants (as defined in (58) ). We assume that whenever there is a collision between Q-balls, they fragment completely. With this assumption we are underestimating the number of Q-balls, and in particular the large Q-giants (hence obtaining an upper bound on the reheat temperature).

Within this approximation, the equation of motion for $n_{Q}$ is given by,

$$
\frac{d n_{Q}}{d t}+3 H n_{Q}=-n_{Q}^{2} \sigma v
$$

with $\sigma$ estimated to be of order the geometric cross-section $\sigma \simeq \pi R^{2}$, which is approximately constant. In the term on the right hand side we have neglected some order one coefficient which is related to the fact that the decay width is proportional to the total number of Q-balls and not just the Q-giants defined in (71). As was shown in section 4.2.2, we may take the initial velocity, which redshifts like $a^{-1}$, to be of order $v_{0} \sim \sqrt{|K|}$. Thus using (44), one finds

$$
n_{Q}(t) \simeq n_{Q}^{0}\left(\frac{a_{0}}{a}\right)^{3}\left[1+\frac{2 \pi \alpha}{5} \frac{n_{Q}^{0}}{m_{\phi}^{3}|K|^{3 / 2}}\left(1-\left(\frac{a_{0}}{a}\right)^{5 / 2}\right)\right]^{-1} \equiv n_{Q}^{0}\left(\frac{a_{0}}{a}\right)^{3} \epsilon_{\mathrm{coll}}(a)
$$

where $n_{Q}^{0} \simeq \epsilon m_{\phi}^{3}|K|^{3 / 2}$ is the initial number density for Q-giants with charge of order $\langle Q\rangle$. We see that collisions become less effective as the universe expands. This is easily understood since, as the number density of the Q-giants drops down, collisions become more rare. Taking again $\alpha \simeq 30$, we learn that collisions reduce the number of Q-giants by roughly two orders of magnitude, namely

$$
\epsilon_{\mathrm{coll}}(a \rightarrow \infty) \gtrsim 10^{-2}
$$

Thus even if we assume that collisions break Q-giants only into $\phi$ gas (without small Qballs), we see that still a few percent of the energy density is left in the Q-giants before the $\phi$ gas decays. Thus we may take,

$$
f_{\text {gas }} \simeq 1-\epsilon_{\mathrm{coll}}(a \rightarrow \infty)
$$

[see eq. (61)].

for fusion, but this will make the Q-giants larger. The cross-section for fusion strongly depends on the phase correlation between colliding Q-giants. Q-giants with a similar phase will tend to merge, while the fusion crosssection for randomly distributed phases is highly suppressed. 
Since we have assumed that the collisions cause the Q-giants to fragment, this is a lower bound on the number of Q-giants. Two additional mechanisms change the survival rate of the Q-giants upwards by a small amount:

1. The field theory binding energy of the $Q$-balls is of order $|K| M_{Q}$, where $M_{Q}$ is the mass of the Q-ball. The kinetic energy is initially of order $M_{Q} v^{2} / 2 \simeq M_{Q} K$. The kinetic energy, however, redshifts rapidly and very quickly there is not enough kinetic energy to overcome the binding energy. This will increase the survival rate of the Q-giants by a factor of order 1 .

2. In the simulations [46] Q-balls often do not fragment upon collision. In fact, for the Q-giants the gravitational binding energy makes them even more resistant to fragmentations since the gravitational energy,

$$
\left|E_{\text {grav }}\right| \simeq \frac{m_{\phi}^{2} Q^{2}}{M_{\mathrm{Pl}}^{2} R} \simeq \frac{10^{4}}{\alpha^{4}} \frac{|K|^{3 / 2} M_{\mathrm{Pl}}^{2}}{m_{\phi}},
$$

is proportional to $Q^{2}$ and is similar in order to the binding energy of the field in flat space (see (48) ) which is

$$
\left|E_{\text {binding }}\right| \simeq|K| m_{\phi} Q \simeq \frac{10^{2}}{\alpha^{2}} \frac{|K|^{3 / 2} M_{\mathrm{Pl}}^{2}}{m_{\phi}} .
$$

$E_{\text {grav }}$ is proportional to $Q^{2}$, which means that large Q-giants are energetically favored over several smaller Q-giants of the same charge. However, for $\alpha \simeq 30$, this effect is rather small.

The conclusion is that by the time the collisions end - shortly after the Q-giants form about $1 \%$ of the total charge and energy is in non-relativistic Q-giants, and the remaining charge and energy is in the $\phi$ gas. Hence, when the gas decays, there is a short period of radiation domination, before the Q-giants become dominant again. As we show in the following sections, given the intermediate temperature, eq. (69), there is enough time between the decay of the gas and the decay of the Q-giants for the Q-giants to take over and dominate of the energy density.

\subsection{Dissociation of Q-Giants}

After the $\phi$-gas decayed, the universe can be thought of as two subsystems composed of Qgiants and radiation. Because the gas never reached thermal equilibrium before decaying, the two systems are not in thermal equilibrium. Nevertheless, the effects of thermal radiation may be important and must be taken into account. We now compute a bound on the temperature outside the Q-giants below which, the Q-giants dissociate. To do so, we briefly repeat the considerations presented in [11]. 
Thermal particles can penetrate Q-giants thereby transferring energy and possibly causing dissociation. The condition to evade dissociation is that the excess energy per unit charge (which was not radiated away) is smaller than the binding energy per unit charge $\delta m \sim|K| m_{\phi}$ :

$$
\left.\frac{\Delta M_{Q}}{Q}\right|_{\delta t_{r}}<\delta m
$$

where $\delta t_{r}$ is the time scale over which the Q-giants radiates its excess energy away. The flux of particles hitting the Q-giant is

$$
\Gamma=\frac{g_{*}(T)}{\pi^{2}} 4 \pi r_{s t}^{2} T^{3}
$$

where $r_{s t}$ is the stopping radius of the Q-giant defined by $g \phi\left(r_{s t}\right) \simeq 3 T$ with $g \simeq \mathcal{O}(1)$ being the coupling constant of $\phi$ to radiation and $g_{*}$ is the number of relativistic degrees of freedom coupled to the Q-giant. Taking the energy per thermal particle transferred to the Q-giant be $\gamma_{T} T$, we get

$$
\frac{d M_{Q}}{d t}=M_{Q} \Gamma=\frac{4 \gamma_{T} g_{*}(T)}{\pi} r_{s t}^{2} T^{4} .
$$

$r_{s t}$ can be easily calculated by using the ansatz (37),

$$
r_{s t}=\beta R, \quad \beta=\log ^{1 / 2}\left(\frac{g \phi_{0}}{3 T}\right) .
$$

Thus, using (39) for $R$ we get,

$$
\frac{d M_{Q}}{d t}=\frac{8 \gamma_{T} g_{*}(T) \beta^{2}}{\pi|K| m_{\phi}^{2}} T^{4}
$$

which, from the inequality (78) and using $\delta t_{r} \simeq 1 / m_{\phi}$ one obtains

$$
T \leq 10^{8}\left(\frac{K}{10^{-2}}\right)^{1 / 2}\left(\frac{200}{g_{*}}\right)^{1 / 4}\left(\frac{m_{\phi}}{10^{3} \mathrm{GeV}}\right)\left(\frac{Q}{10^{28}}\right)^{1 / 4} \mathrm{GeV},
$$

where we have taken $\gamma_{T} \simeq 1$ and $\beta \simeq 4$.

In [11] this inequality is used as a bound on the reheat temperature. Here, we use it as a bound on the maximal temperature outside the Q-giants. It is therefore immediately clear that the radiation after the decay of the gas cannot dissociate the Q-giants. Below, we also investigate the reheating process during the decay of the Q-giants themselves. We show that dissociation does not play a role at that stage either.

\subsection{Reheating}

In general Q-balls can decay into fermions and bosons through the surface where these particles are light [45, 11]. The decay into fermions was found to be bounded by [45]

$$
\frac{d Q}{d t d A} \leq \frac{\omega_{0}^{3}}{192 \pi^{2}}
$$


with $\omega_{0}^{2}=\omega^{2}-m_{\phi}^{2}(1+K) \simeq 3 m_{\phi}^{2}|K|$, whereas the decay into bosons, as was shown in [1], is enhanced by a factor $f_{s} \simeq 170 /|K|^{1 / 2} .{ }^{14}$ From the above, one obtains an upper bound on the decay width of the Q-giants ${ }^{15}$

$$
\Gamma_{Q}=\frac{1}{E_{Q}} \frac{d E_{Q}}{d t} \leq \Gamma_{Q}^{\max } \simeq \frac{f_{s}}{24 \pi} \frac{\sqrt{|K|} m_{\phi}}{Q} .
$$

Let us now evaluate the temperature outside during and at the end of the reheating process. To do so, we solve the Boltzmann equations,

$$
\begin{aligned}
\frac{d \rho_{\gamma}}{d t}+4 H \rho_{\gamma} & =\int_{Q_{\min }}^{Q_{\max }} d Q f(t, Q) \Gamma_{Q}^{\max } M(Q), \\
\frac{\partial f(t, Q)}{\partial t}+3 H f(t, Q) & =Q \Gamma_{Q}^{\max } \frac{\partial f(t, Q)}{\partial Q} .
\end{aligned}
$$

where $f(t, Q)=\int d^{3} v f(t, Q, v)$ is the time-dependent density distribution function for a Q-ball with charge $\mathrm{Q}, \rho_{\gamma}$ is the energy density stored in radiation and $M(Q) \simeq Q m_{\phi}$ is the mass of the Q-ball (see eq. (42)). The last term in eq. (87) is a transport term in Q-space, which takes into account the fact that as the Q-giant decays, it loses its charge gradually. The solution for (87) is given by,

$$
f(t, Q)=\left(\frac{a}{a_{0}}\right)^{-3} f\left(t_{0}, Q+\hat{\Gamma}_{Q}^{\max }\left(t-t_{0}\right)\right)
$$

where $\hat{\Gamma}_{Q}^{\max }=Q \Gamma_{Q}^{\max } \simeq m_{\phi}$ is independent of $Q$. Thus, $f(t, Q)$ is simply the initial Gaussian distribution with a shift of its central value, $\langle Q\rangle \rightarrow\langle Q\rangle-\hat{\Gamma}_{Q}^{\max }\left(t-t_{0}\right)$. This solution is easy to understand. For small $t, t-t_{0} \ll\langle Q\rangle / \hat{\Gamma}_{Q}^{\max }=\left(\Gamma_{\langle Q\rangle}^{\max }\right)^{-1}$, the distribution is approximately constant and the Q-giants redshift like matter. At $H^{-1}=t-t_{0}=\left(\Gamma_{\langle Q\rangle}^{\max }\right)^{-1}$ the center of the distribution becomes negative where the solution is no longer valid. At this point, the Q-giants decay instantaneously transferring the energy to radiation.

Next, we solve for the energy density in radiation. The solution to (86) is

$$
\rho_{\gamma}=\left(\frac{a(t)}{a_{0}}\right)^{-4} \int_{t_{i}}^{t} d t^{\prime}\left(\frac{a\left(t^{\prime}\right)}{a_{0}}\right)^{4} \int_{Q_{\min }}^{Q_{\max }} d Q f\left(t^{\prime}, Q\right) \Gamma_{Q}^{\max } M(Q) .
$$

Using the above approximation of constant $f(t, Q)$, we get for $t-t_{0} \ll\left(\Gamma_{\langle Q\rangle}^{\max }\right)^{-1}$,

$$
\rho_{\gamma} \simeq \frac{3(1-\epsilon) m_{\phi}^{5}|K|^{3 / 2}}{5 H_{0}}\left(\frac{a}{a_{0}}\right)^{-4}\left[\left(\frac{a}{a_{0}}\right)^{5 / 2}-1\right]
$$

\footnotetext{
${ }^{14}$ Note that the actual decay rate for the Q-giant scenarios is much smaller initially until the gas between the Q-giants decay.

${ }^{15}$ The reader should note that finite temperature decay, as was computed in [4] is not relevant in our scenario since the Q-giants are very weakly coupled to the light particles.
} 
where we took $Q_{\min }=0$ and $Q_{\max }=\infty$, and $H_{0}$ is given in (44). Thus at the transient time, radiation reheats to a maximal temperature,

$$
T_{\max } \simeq 10^{3}\left(\frac{m_{\phi}}{10^{3} \mathrm{GeV}}\right)\left(\frac{\alpha}{30}\right)^{1 / 4}\left(\frac{|K|}{10^{-2}}\right)^{1 / 8}\left(\frac{g_{*}}{200}\right)^{-1 / 4} \mathrm{GeV}
$$

and redshifts like $a^{-3 / 2}$ until $t \simeq\left(\Gamma_{\langle Q\rangle}^{\max }\right)^{-1}$ when the Q-giants decay completely, reheating the universe to

$$
T_{\text {reheat }}=\left(\frac{m_{\phi} M_{\mathrm{Pl}}}{g_{*}^{1 / 2} Q}\right)^{1 / 2}=10^{-4}\left(\frac{m_{\phi}}{10^{3} \mathrm{GeV}}\right)^{1 / 2}\left(\frac{Q}{10^{28}}\right)^{-1 / 2}\left(\frac{g_{*}}{200}\right)^{-1 / 4} \mathrm{GeV} .
$$

Once the Q-giants decayed, radiation redshifts like $a^{-4}$ as usual.

As was shown in 4.3, the intermediate reheat temperature from the decay of the gas is some nine orders of magnitude above the reheat temperature of the Q-giants. This, as we advocated above, is sufficient to allow the Q-giants to regain domination over the energy density, lost due to collisions. Furthermore, given the bound on dissociation, eq. (83), we thus find that during the reheating process, Q-giants are not dissociated and the final reheating temperature is very low due to their large charge. Hence, the Q-giants dominate the energy density too long, leading to a too low reheating temperature for a successful conventional big bang nucleosynthesis required in the standard cosmological scenario. We are therefore led to conclude that negative pressure flat directions cannot play a role in modular inflation models.

\section{Positive Pressure Flat Directions and Primordial Black Holes}

We now turn to flat directions which, due to RG running, generate positive pressure, namely, $K>0$. Such flat directions do not fragment to form Q-balls, but rather generate a finite Jeans scale which prevents the formation of primordial black hole after inflation [4]. Indeed since typically the modulus that drives inflation is weakly coupled, there is a long stage of matter domination after inflation which can generate large density perturbations and lead to the creation of BHs [19]. It is interesting that within the MSSM, the only direction which is not charged under B-L and which has positive pressure is the $H_{u} H_{d}$ direction.

Consider again the potential (30), only this time with positive $K$. As is shown in Appendix A. the equation of state is approximately given by,

$$
P=\frac{K}{K+2} \rho \simeq \frac{K}{2} \rho
$$


and the Jeans scale is therefore

$$
\lambda_{J}=\frac{K}{2} H^{-1}
$$

At scales smaller than $\lambda_{J}$ pressure prevents growth of perturbations and such perturbations oscillate. Thus whether or not BHs can form, depends on the value of $K$ at the time density perturbations becomes non-linear. $K$ is given by

$$
K=\left.\frac{1}{m_{\phi}^{2}} \frac{\partial m_{\phi}^{2}}{\partial t}\right|_{t=\log \left(|\phi|^{2} / M^{2}\right)}
$$

with $t=\log \left(\mu^{2} / M^{2}\right)$ and can therefore be extracted from the RGE's of a given model. In what follows, we assume the MSSM up to the GUT scale. Our strategy is to find the Jean's scale at the time where $\delta \rho / \rho$ is of order one and compare it with the scale of the fluctuations. If the Jean's scale is larger we are assured that no Q-balls or BHs can form.

Assuming $\delta \rho / \rho \simeq 5 \times 10^{-4}$ for scales of order the horizon at the end of inflation, and using the fact that during matter domination, perturbations grow like $a$ while $\phi$ redshift like $a^{-3 / 2}$, we find

$$
\mu=|\phi|_{\delta \rho / \rho=1}=\frac{M_{\mathrm{Pl}}}{\left(5 \times 10^{-4}\right)^{-3 / 2}} \simeq 10^{13} \mathrm{GeV} .
$$

On the other hand, a density fluctuation of size $\lambda_{\mathrm{inf}} \sim H_{\mathrm{inf}}^{-1}$ at the end of inflation becomes when $\delta \rho / \rho=1$,

$$
\left.\lambda\right|_{\delta \rho / \rho=1}=a^{-1 / 2} H^{-1} \simeq 2.2 \times 10^{-2} H^{-1} .
$$

Thus to a-priori eliminate the possibility of $\mathrm{BH}$ formation we must look for a flat direction of the MSSM with $K / 2 \gtrsim 2.2 \times 10^{-2}$.

The RGE's for the MSSM are given in [49]. For the particular case of $H_{u} H_{d}$ flat direction, one finds ${ }^{16} K / 2 \simeq 2.3 \times 10^{-2}$. We can therefore conclude that no primordial BHs can form if the $H_{u} H_{d}$ direction plays the role of the modulus in inflation. Moreover, it is the only possible direction in the MSSM. Before structure can form, the modulus must decay and reheat the MSSM. After that, structure formation can follow as usual, in the radiation dominated universe.

Note that we have imposed a very strong restriction, which is fortunately fulfilled in the MSSM. It could well be that $\delta \rho / \rho \simeq 1$ is permissible, in which the universe undergoes a period of structure formation by $\phi$ particles, similar to cold dark gas. It will be interesting to explore this possibility further.

\footnotetext{
${ }^{16}$ We thank Guy Englehard for enabling us to use his Mathematica code for the RGE's.
} 


\section{Discussion and Caveats}

In this section, we briefly detail what would go wrong with cosmological scenarios in which the negative pressure flat directions survive and argue that thermal inflation [23, 24, 25] 17 may change the conclusions. However, we present reasons for the belief that invoking thermal inflation in the present context requires fine tuning.

With Q-giants surviving a long time, big bang nucleosynthesis would be severely disrupted since the historical sequence of radiation domination (RD) and matter domination (MD) periods will be modified from the standard scenario of

$$
\text { inflation }+\mathrm{MD} \rightarrow \mathrm{RD}(\mathrm{BBN}) \rightarrow \mathrm{MD} \rightarrow \text { today }
$$

to

$$
\text { inflation }+\mathrm{MD} \rightarrow \mathrm{RD} \rightarrow \mathrm{MD}(\mathrm{BBN}) \rightarrow \mathrm{RD} \rightarrow \mathrm{MD} \rightarrow \text { today }
$$

or

inflation $+\mathrm{MD} \rightarrow \mathrm{RD} \rightarrow$ thermal inflation $+\mathrm{MD} \rightarrow \mathrm{RD}(\mathrm{BBN}) \rightarrow \mathrm{MD} \rightarrow \mathrm{RD} \rightarrow \mathrm{MD} \rightarrow$ today.

Eq. (99) depicts the situation in which there is no thermal inflation, while Eq. (100) depicts the situation in which there is thermal inflation to dilute possibly too large baryon number (note that we have included any possible additional period of matter domination between thermal inflation and radiation domination following from flaton decay). The label "BBN" marks the period during which nucleosynthesis occurs. A successful nucleosynthesis is impossible by the time of last RD periods labeled in Eqs. (99) and (100) since the maximal reheating temperature of $0.1 \mathrm{MeV}$ during those time periods is smaller than $\sim 10 \mathrm{MeV}$ required for chemical equilibrium initial conditions or $\sim 1 \mathrm{MeV}$ when neutrons drop out of equilibrium [50].

Clearly, without thermal inflation BBN occurs during matter domination and thus the existence of Q-giants is excluded. One can also show that the baryon-to-entropy ratio during $\mathrm{BBN}$ in that case does not allow for a successful production of the light elements. We hence consider the scenario depicted by Eq. (100) in which one invokes a short period of thermal inflation [23, 24, 25]. Assume that we have the flaton, $\varphi$ (not to be confused with $\phi$ ), at some VEV, $M$, initially at its true vacuum. Once the gas decays, reheating the outer region of the Q-giants to a maximal temperature of $T_{\text {begin }}=T_{\text {inter }}=10^{5} \mathrm{GeV}$, thermal corrections drive $\varphi$ to the origin, and thermal inflation begins at roughly that temperature. It ends when

\footnotetext{
${ }^{17}$ We thank Ewan Stewart for drawing our attention to this issue.
} 
$T_{\text {end }}=m_{\varphi} \simeq 10^{3} \mathrm{GeV}$ and thus there is at most 5 e-folds of thermal inflation, which can reduce the energy density in the Q-giants by $10^{-6}$.

The energy density during thermal inflation is of order $V_{0}=m_{\varphi}^{2} M^{2}$. Since the flaton stores no energy when it sits in the true minimum, the total energy density once the gas decays is of order $g_{*} T_{\text {inter }}^{4}$ and therefore $V_{0}$ must be smaller, giving,

$$
M \lesssim 10^{8}\left(\frac{m_{\varphi}}{10^{3} \mathrm{GeV}}\right)^{-1}\left(\frac{g_{*}}{200}\right)^{1 / 2} \mathrm{GeV}
$$

The above can be understood in a different way, by noting that at $M, \varphi$ is coupled to radiation through massive particles with mass $M$. Thus corrections to its mass are expected to be of order $T^{4} / M^{2}$ and therefore, for these corrections to drive it to zero, (101) must hold.

Within the context of gravity mediated supersymmetry breaking one can argue that such a requirement from thermal inflation is difficult to achieve 23. Indeed, the flaton is expected to have some $\mathbb{Z}_{n}$ symmetry $(n>2)$ with a superpotential,

$$
W=\frac{\varphi^{n}}{M_{\mathrm{Pl}}^{n-3}}+\ldots
$$

Taking into account soft breaking mass $-m_{3 / 2}^{2} \varphi^{2}$ and A-terms $m_{3 / 2} W$ one finds the minimum of the potential to be at

$$
M=\langle\varphi\rangle \simeq M_{\mathrm{Pl}}\left(\frac{m_{3 / 2}}{M_{\mathrm{Pl}}}\right)^{1 /(n-2)} .
$$

Hence the only value of $M$ which is compatible with (101) is $M=m_{3 / 2} \simeq 10^{3} \mathrm{GeV}$ for $n=3$. However, in such a case, thermal inflation begins only at $T_{\text {begin }}=10^{3}=T_{\text {end }}$ and so there is no time for thermal inflation to dilute the Q-giants. Thus invoking a period of thermal inflation in this scenario requires fine tuning. Nevertheless, for completeness, let us suppose that one is able to produce such an epoch and see what are the requirements for thermal inflation to sufficiently dilute the Q-giants.

At the end of thermal inflation, assuming perturbative decay of the flaton, the energy density in radiation is of order $\rho_{\gamma}^{\text {flat-decay }}\left(T_{\text {flat }}\right)=\gamma m_{\varphi}^{2} M^{2}$ where $\gamma$ is a factor coming from the volume dilution during the coherent oscillation of the flaton and $10^{-2} \mathrm{GeV}<T_{\text {flat }}<10^{3} \mathrm{GeV}$ is the flaton's reheating temperature (note that the lower bound on the flaton decay temperature comes from the requirement of successful BBN). On the other hand, the energy density in Q-giants is (including collisions - see section [5.1),

$$
\rho_{Q}\left(T_{\text {flat }}\right) \simeq 10^{-8} \gamma g_{*}\left(T_{\text {begin }}\right) T_{\text {begin }}^{4} .
$$


After the flaton decays, in principle, there could occur a baryogenesis scenario and then BBN can commence afterwards. Now, for BBN not to be disrupted, we must have radiation domination during that time. This implies,

$$
M \gtrsim 10^{6.5}\left(\frac{m_{\varphi}}{10^{3} \mathrm{GeV}}\right)^{-1}\left(\frac{T_{\text {flat }}}{10^{3} \mathrm{GeV}}\right)^{1 / 2}\left(\frac{g_{*}}{200}\right)^{1 / 2} \mathrm{GeV} .
$$

The lower bound decreases for smaller $T_{\text {flat }}$ because the flaton decay radiation energy density dilutes faster with the scale factor than when the flaton energy density is stored in the form of coherent oscillations. In the limit that the flaton never decays but remains in coherent oscillations, since the Q-giants energy density dilutes the same way as that of the flaton, they never come to dominate the energy density. Eqs. (101) and (105) mean that in principle, thermal inflation can allow those flat directions not carrying B to survive.

If the Q-giants carry $\mathrm{B}$ charge ${ }^{18}$ the situation is more constrained. In order not to produce too much baryon number, the Q-giants must never dominate the energy density. In such a case it is simple to compute the baryon asymmetry at the time of Q-giant decay, $T_{\text {reheat }} \simeq 10^{-4}$. Indeed, at $T_{\text {reheat }}$,

$$
\rho_{Q}\left(T_{\text {reheat }}\right) \simeq \rho_{Q}\left(T_{\text {flat }}\right)\left(\frac{T_{\text {reheat }}}{T_{\text {flat }}}\right)^{3}
$$

while,

$$
\rho_{\gamma}\left(T_{\text {flat }}\right) \simeq \gamma m_{\varphi}^{2} M^{2}\left(\frac{T_{\text {reheat }}}{T_{\text {flat }}}\right)^{4}
$$

Hence using $n_{B} / s \simeq\left(\rho_{Q} / m_{\phi}\right) / \rho_{\gamma}^{3 / 4}$ and by demanding that the baryon-to-entropy ratio so produced is smaller than the measured $10^{-10}$, one finds

$$
M \gtrsim 10^{9}\left(\frac{m_{\varphi}}{10^{3} \mathrm{GeV}}\right)^{-3 / 2}\left(\frac{T_{\text {flat }}}{10^{3} \mathrm{GeV}}\right)^{1 / 2}\left(\frac{g_{*}}{200}\right)^{1 / 2} \mathrm{GeV}
$$

Thus thermal inflation can, in principle, dilute the baryon number produced by Q-giants decay, if its reheat temperature is between $10^{-2} \mathrm{GeV} \leq T_{\text {flat }} \leq 10 \mathrm{GeV}$.

Finally, consider the flat direction with positive pressure such that no Q-giants form. In that case, thermal inflation can acceptably dilute the baryon number density if

$$
M \gtrsim 10^{10}\left(\frac{T_{\mathrm{RH}}}{10^{5} \mathrm{GeV}}\right)^{1 / 2}\left(\frac{T_{\text {flat }}}{10^{3} \mathrm{GeV}}\right)^{1 / 2}\left(\frac{g_{* S}\left(T_{\mathrm{RH}}\right)}{200}\right)^{1 / 2} \mathrm{GeV} .
$$

\footnotetext{
${ }^{18}$ Note that phenomenologically, it is more appropriate to classify the scenarios according to baryon number since the observational constraints on lepton number is very weak [51].
} 
where $T_{\mathrm{RH}} \leq 10^{5} \mathrm{GeV}$ is the reheating temperature after inflation ends ${ }^{19}$ and just as before, $T_{\text {flat }}$ corresponds to the temperature at which the flaton decays. For sufficiently small $T_{\mathrm{RH}}$ and $T_{\text {flat }}$ one can dilute the produced baryon number to an acceptable value. Hence, the flat direction $H_{u} L$ for which $K>0$ can survive with sufficient engineering of thermal inflation. (Note that if the reheating temperature is sufficiently low, sphalerons may be sufficiently suppressed to forbid the conversion of the lepton number into baryon number. In that case, no further dilution from thermal inflation may be necessary.)

To conclude this section, we point out that while thermal inflation can, for a narrow window of the reheating temperature, dilute the Q-giants sufficiently thus evading the constraints considered in the previous sections, such a theory requires fine tuning and so seems to be improbable. The same conclusion holds for positive pressure flat directions. Finally, even for the flat directions that are generically "excluded," extremely unlikely fine tuning of initial conditions (such that no charge is produced) may allow them to be viable.

\section{Conclusions}

It has long been realized that negative pressure flat directions fragment into Q-balls which are rather stable and may survive the EW phase transition. In this paper, we have considered the evolution of such flat directions that are assumed to play a role in inflation, and which therefore have VEVs of order the Planck scale at the end of inflation and which dominate the energy density at that time. Such flat directions are found, for example, in hybrid inflation models. We have shown that within the framework of gravity mediated supersymmetry breaking, these flat directions fragment into very large "Q-giants" which survive nucleosynthesis and are therefore excluded cosmologically.

The small decay width of the Q-giants is directly related to its large charge. However, thermal effects may break and dissociate the Q-giants long before they decay. Indeed, a new effect which was not considered in previous works is the formation and decay of a gas outside the Q-giants. During the fragmentation of a flat direction, $\phi$, not only Q-giants are formed, but also $\phi$ particles in the form of a non-thermal gas. Although, the majority of the charge is stored inside the Q-giants, there is an order one fraction of energy density stored in the gas. While outside the $\mathrm{Q}$-giants $\langle\phi\rangle$ vanishes, the naive expectation, that the $\phi$-gas decays very

\footnotetext{
${ }^{19}$ Note that the finite density effect applies independently of whether there is negative pressure or not. Note further that the upper bound of $10^{5} \mathrm{GeV}$ on the temperature of the universe is quite robust in such models where the modulus controls the energy density.
} 
quickly and reheats the universe to high temperature which would dissociate the Q-giants, is wrong. The reason is that $\left\langle\phi^{\dagger} \phi\right\rangle$ generates large masses to the particles to which it couples, and therefore its decay is mediated through these particles with masses of order $\left\langle\phi^{\dagger} \phi\right\rangle$. One finds the intermediate temperature outside the Q-giants, once the gas decays to be,

$$
T_{\text {inter }} \simeq 10^{5} \mathrm{GeV}
$$

which is much less than the dissociation temperature, $T_{\text {diss }} \simeq 10^{8} \mathrm{GeV}$. Thus the temperature outside the Q-giants is never high enough to dissociate them.

Taking collisions into account, we have also shown that by the time the gas decays it dominates the energy density and therefore there is a short period of radiation domination, before the Q-giants take control over the energy density until they decay and reheat the universe to the temperature

$$
T_{\text {reheat }} \lesssim 10^{-4} \mathrm{GeV}
$$

which is generically too low of a reheating temperature to be compatible with cosmology.

Hence, without further entropy-releasing mechanisms, negative pressure flat direction scenarios are not viable cosmological scenarios. As an example of entropy-releasing mechanism, we have considered thermal inflation and found that such a scenario requires fine tuning. Furthermore, such period of inflation must be properly engineered, with only a narrow window for the reheating temperature that allows these flat directions to become viable again.

The only flat directions that do not suffer from too low reheating temperature without thermal inflation are positive pressure flat directions. Hence, within the MSSM, the only possible directions are $H_{u} H_{d}$ and $H_{u} L$. Since the latter is charged under B-L and if the reheating temperature is large enough to produce baryon number, it requires a dilution mechanism like thermal inflation (which again requires fine tuning). Finally, we point out that these directions prevent the formation of primordial BHs due to a generation of a finite Jeans scale.

\section{Acknowledgement}

We would like to thank M. Dine, N. Itzhaki, A. Kusenko, D. Kutasov, and L. Wang for useful discussions. We also thank Y. Nir and L. Everett for comments on the manuscript. The work of MB is supported in part by the Israel Science Foundation, by the Braun-Roger-Siegl foundation, by the European network HPRN-CT-2000-00122, by a grant from the G.I.F. (the German- 
Israeli Foundation for Scientific Research and Development), by the Minerva Foundation, by the Einstein Center for Theoretical Physics and the by Blumenstein foundation.

\section{A The Virial Theorem and Equation of State}

We present here a simple derivation to the pressure generated by a scalar condensate oscillating in the potential (30) which can also be approximated by

$$
U=m_{\phi}^{2} \frac{|\phi|^{2 K+2}}{M^{2 K}} .
$$

This result was first derived in [10].

To do so we consider an alternative version of the virial theorem. Consider the quantity,

$$
\langle\dot{F}\rangle=\left\langle\frac{d}{d t}\left(\phi \dot{\phi}^{\dagger}+\dot{\phi} \phi^{\dagger}\right)\right\rangle
$$

where the average is taken with respect to time. Hence, as long as the motions are bounded, we have

$$
\begin{aligned}
\langle\dot{F}\rangle & =\lim _{T \rightarrow \infty} \frac{1}{T} \int_{0}^{T} \dot{F} d t \\
& =\lim _{T \rightarrow \infty} \frac{1}{T}[F(T)-F(0)]=0
\end{aligned}
$$

Thus we can write,

$$
\begin{aligned}
0 & =\left\langle 2|\dot{\phi}|+\phi \ddot{\phi}^{\dagger}+\ddot{\phi} \phi^{\dagger}\right\rangle \\
& =\left\langle 2|\dot{\phi}|-\phi \partial_{\phi} U-\phi^{\dagger} \partial_{\phi^{\dagger}} U\right\rangle \\
& =\left\langle 2|\dot{\phi}|-2 m_{\phi}^{2}|\phi|^{2}\left(1+K+K \ln \frac{|\phi|^{2}}{M^{2}}\right)\right\rangle
\end{aligned}
$$

where we have used the potential (30) and ignored the expansion of the universe. This is justified assuming the oscillation period is much smaller than $H^{-1}$. We therefore find,

$$
\langle|\dot{\phi}|\rangle=m_{\phi}^{2}\left\langle|\phi|^{2}\left(1+K+K \ln \frac{|\phi|^{2}}{M^{2}}\right)\right\rangle .
$$

Next, we have,

$$
\begin{aligned}
\rho & =\left\langle|\dot{\phi}|^{2}+U\right\rangle=m_{\phi}^{2}\left\langle|\phi|^{2}\left(2+K+2 K \ln \frac{|\phi|^{2}}{M^{2}}\right)\right\rangle, \\
P & =\left\langle|\dot{\phi}|^{2}-U\right\rangle=m_{\phi}^{2} K\left\langle|\phi|^{2}\right\rangle
\end{aligned}
$$


which gives us

$$
\frac{P}{\rho}=\frac{K}{2+K+\left\langle 2 K|\phi|^{2} \ln \frac{|\phi|^{2}}{M^{2}}\right\rangle /\left\langle|\phi|^{2}\right\rangle} .
$$

Similarly, using the potential (112) on finds,

$$
\frac{P}{\rho}=\frac{K}{2+K}
$$

\section{References}

[1] M. Sasaki and E. D. Stewart, Prog. Theor. Phys. 95, 71 (1996) arXiv:astro-ph/9507001.

[2] D. J. H. Chung, L. L. Everett, G. L. Kane, S. F. King, J. Lykken and L. T. Wang, Phys. Rept. 407, 1 (2005) arXiv:hep-ph/0312378.

[3] T. D. Lee and Y. Pang, Phys. Rept. 221, 251 (1992).

[4] S. R. Coleman, Nucl. Phys. B 262, 263 (1985) [Erratum-ibid. B 269, 744 (1986)].

[5] K. M. Lee, J. A. Stein-Schabes, R. Watkins and L. M. Widrow, Phys. Rev. D 39, 1665 (1989).

[6] A. Kusenko, Phys. Lett. B 405, 108 (1997) arXiv:hep-ph/9704273.

[7] G. R. Dvali, A. Kusenko and M. E. Shaposhnikov, Phys. Lett. B 417, 99 (1998) arXiv:hep-ph/9707423.

[8] K. Enqvist and J. McDonald, Phys. Lett. B 425, 309 (1998) arXiv:hep-ph/9711514.

[9] A. Kusenko and M. E. Shaposhnikov, Phys. Lett. B 418, 46 (1998) arXiv:hep-ph/9709492.

[10] M. S. Turner, Phys. Rev. D 28, 1243 (1983).

[11] K. Enqvist and J. McDonald, Nucl. Phys. B 538, 321 (1999) arXiv:hep-ph/9803380.

[12] K. Enqvist, A. Jokinen and J. McDonald, Phys. Lett. B 483, 191 (2000) arXiv:hep-ph/0004050.

[13] S. Kasuya and M. Kawasaki, Phys. Rev. D 62, 023512 (2000) arXiv:hep-ph/0002285.

[14] K. Enqvist and A. Mazumdar, Phys. Rept. 380, 99 (2003) arXiv:hep-ph/0209244.

[15] L. Randall, M. Soljacic and A. H. Guth, Nucl. Phys. B 472, 377 (1996) arXiv:hep-ph/9512439; L. Randall, M. Soljacic and A. H. Guth, arXiv:hep-ph/9601296. 
[16] M. Dine, L. Randall and S. Thomas, Phys. Rev. Lett. 75, 398 (1995) arXiv:hep-ph/9503303.

[17] M. Dine, L. Randall and S. Thomas, Nucl. Phys. B 458, 291 (1996) arXiv:hep-ph/9507453.

[18] A. D. Linde, Phys. Lett. B 259, 38 (1991); A. D. Linde, Phys. Rev. D 49, 748 (1994) arXiv:astro-ph/9307002.

[19] M. Berkooz, M. Dine and T. Volansky, Phys. Rev. D 71, 103502 (2005) arXiv:hep-ph/0409226.

[20] P. Binetruy and G. R. Dvali, Phys. Lett. B 388, 241 (1996) arXiv:hep-ph/9606342.

[21] E. Halyo, Phys. Lett. B 387, 43 (1996) arXiv:hep-ph/9606423.

[22] E. D. Stewart, Phys. Lett. B 391, 34 (1997) arXiv:hep-ph/9606241; E. D. Stewart, Phys. Rev. D 56, 2019 (1997) arXiv:hep-ph/9703232.

[23] D. H. Lyth and E. D. Stewart, Phys. Rev. D 53, 1784 (1996) arXiv:hep-ph/9510204.

[24] E. D. Stewart, M. Kawasaki and T. Yanagida, Phys. Rev. D 54, 6032 (1996) arXiv:hep-ph/9603324.

[25] T. Asaka and M. Kawasaki, Phys. Rev. D 60, 123509 (1999) arXiv:hep-ph/9905467.

[26] D. H. Lyth, Phys. Rev. Lett. 78, 1861 (1997) arXiv:hep-ph/9606387.

[27] A. D. Linde, Phys. Lett. B 129, 177 (1983).

[28] E. J. Copeland, A. R. Liddle, D. H. Lyth, E. D. Stewart and D. Wands, Phys. Rev. D 49, 6410 (1994) arXiv:astro-ph/9401011.

[29] E. D. Stewart, Phys. Rev. D 51, 6847 (1995) arXiv:hep-ph/9405389.

[30] D. H. Lyth, Phys. Lett. B 419, 57 (1998) arXiv:hep-ph/9710347.

[31] T. Gherghetta, C. F. Kolda and S. P. Martin, Nucl. Phys. B 468, 37 (1996) arXiv:hep-ph/9510370.

[32] H. P. Nilles, M. Srednicki and D. Wyler, Phys. Lett. B 120, 346 (1983); J. P. Derendinger and C. A. Savoy, Nucl. Phys. B 237, 307 (1984); M. Drees, Int. J. Mod. Phys. A 4, 3635 (1989); J. R. Ellis, J. F. Gunion, H. E. Haber, L. Roszkowski and F. Zwirner, Phys. Rev. D 39, 844 (1989). 
[33] E. W. Kolb, A. Notari and A. Riotto, Phys. Rev. D 68, 123505 (2003) arXiv:hep-ph/0307241.

[34] J. Yokoyama, Phys. Rev. D 70, 103511 (2004) arXiv:hep-ph/0406072.

[35] T. Multamaki and I. Vilja, Nucl. Phys. B 574, 130 (2000) arXiv:hep-ph/9908446.

[36] S. Kasuya and M. Kawasaki, Phys. Rev. D 64, 123515 (2001) arXiv:hep-ph/0106119.

[37] I. Affleck and M. Dine, Nucl. Phys. B 249, 361 (1985).

[38] M. Carena, M. Quiros and C. E. M. Wagner, Phys. Lett. B 380, 81 (1996) arXiv:hep-ph/9603420.

[39] G. R. Farrar and M. Losada, Phys. Lett. B 406, 60 (1997) arXiv:hep-ph/9612346.

[40] B. de Carlos and J. R. Espinosa, Nucl. Phys. B 503, 24 (1997) arXiv:hep-ph/9703212.

[41] M. Laine and K. Rummukainen, Nucl. Phys. B 597, 23 (2001) arXiv:hep-lat/0009025.

[42] M. Berkooz, Y. Nir and T. Volansky, arXiv:hep-ph/0401012.

[43] L. J. Hall, H. Murayama and G. Perez, arXiv:hep-ph/0504248.

[44] A. Menon, D. E. Morrissey and C. E. M. Wagner, arXiv:hep-ph/0404184.

[45] A. G. Cohen, S. R. Coleman, H. Georgi and A. Manohar, Nucl. Phys. B 272, 301 (1986).

[46] T. Multamaki and I. Vilja, Phys. Lett. B 482, 161 (2000) arXiv:hep-ph/0003270.

[47] M. Laine and M. E. Shaposhnikov, Nucl. Phys. B 532, 376 (1998) arXiv:hep-ph/9804237.

[48] B. J. Carr, J. H. Gilbert and J. E. Lidsey, Phys. Rev. D 50, 4853 (1994) arXiv:astro-ph/9405027.

[49] S. P. Martin and M. T. Vaughn, Phys. Rev. D 50, 2282 (1994) arXiv:hep-ph/9311340.

[50] E. W. Kolb and M. S. Turner, The Early Universe, Redwood City, USA: Addison-Wesley (1990) (Frontiers in physics, 69).

[51] A. D. Dolgov, Phys. Rept. 370, 333 (2002) arXiv:hep-ph/0202122. 